\title{
Supporting Information for: Group Contribution and Machine Learning Approaches to Predict Abraham Solute Parameters, Solvation Free Energy, and Solvation Enthalpy
}

\author{
Yunsie Chung, ${ }^{\dagger}$ Florence H. Vermeire,$^{\dagger}$ Haoyang $\mathrm{Wu},{ }^{\dagger}$ Pierre J. Walker, ${ }^{\dagger, \ddagger}$ \\ Michael H. Abraham, ${ }^{\top}, \S$ and William H. Green*,† \\ ${ }^{\dagger}$ Department of Chemical Engineering, Massachusetts Institute of Technology, Cambridge, \\ MA, 02139, U.S.A \\ ${ }^{\ddagger}$ Department of Chemical Engineering, Imperial College London, SW7 2AZ, United \\ Kingdom \\ `Department of Chemistry, University College London, 20 Gordon Street, London WC1H \\ OAJ, United Kingdom \\ $\S$ deceased \\ E-mail: whgreen@mit.edu
}

\section{Data collection and compilation}

The solute and solvent information in the collected databases are given in various representations, such as CAS numbers, InChI, ${ }^{1}$ SMILES, chemical names, and 3D coordinates. The data are standardized by converting the given identifiers to both SMILES and InChI strings using PubChemPy ${ }^{2]}, \mathrm{CIRPy}^{3}, \mathrm{RDKit}^{4}$, and $\mathrm{ARC}^{[5}$. All available chemical identifiers are converted and the resulting InChI strings are compared to eliminate incorrect or ambiguous naming. The stereochemistry of the compounds is encoded in SMILES and InChI if it is specified; otherwise, the compound is represented only based on atom connectivity in SMILES and InChI strings. The non-standard InChI containing a fixed-hydrogen layer is used instead of the standard InChI to distinguish tautomers. Those InChIs are used as unique identifiers to identify different solutes or solute-solvent pairs in the data sets. When multiple data points are found for the same solute or solute-solvent pair, the mean values of the Abraham solute parameters, $\Delta G_{\text {solv }}(298 \mathrm{~K})$, or $\Delta H_{\text {solv }}(298 \mathrm{~K})$ are used and the standard deviations are calculated. No data are removed based on the standard deviations as most of the data are found to have relatively low standard deviations.

\section{Comparison of the LSER estimates and experimental data}

Based on the collected data sets, the errors associated with the LSER are evaluated prior to the construction of the prediction models. Solvation free energies and enthalpies are 
calculated from the Abraham and Mintz LSERs using the experimental solute parameters (SoluteDB) and the fitted solvent parameters, and the calculated values are compared to the dGsolvDB and dHsolvDB data sets. The parity plots and errors are shown in Figure [S1. It can be seen that the LSER has small $\Delta G_{\text {solv }}(298 \mathrm{~K})$ error and relatively higher $\Delta H_{\text {solv }}(298 \mathrm{~K})$ error. These errors are due to both the aleatoric uncertainty of the experimental data and the fact that the linearity of the LSER cannot capture the non-linear relationship between the solute parameters and solvation energy/enthalpy. Since the LSER estimates of both solvation free energy and enthalpy in Figure S1 are calculated using the same experimental solute parameters, it is likely that the model error associated with the Mintz LSER is higher than that of the Abraham LSER. It is also possible that the solvation enthalpy data (dHsolvDB) have higher experimental uncertainty than the solvation energy data (dGsolvDB $x$ ), but we are unable to assess the experimental uncertainties of the data sets used in this work as the majority of solvent-solute pairs only have a single data point. The SoluteGC and SoluteML models that predict $\Delta G_{\text {solv }}(298 \mathrm{~K})$ and $\Delta H_{\text {solv }}(298 \mathrm{~K})$ through the LSER will, at their best performance, have these underlying errors when compared to the $\mathrm{dGsolvDB} x$ and $\mathrm{dHsolvDB}$ data sets.
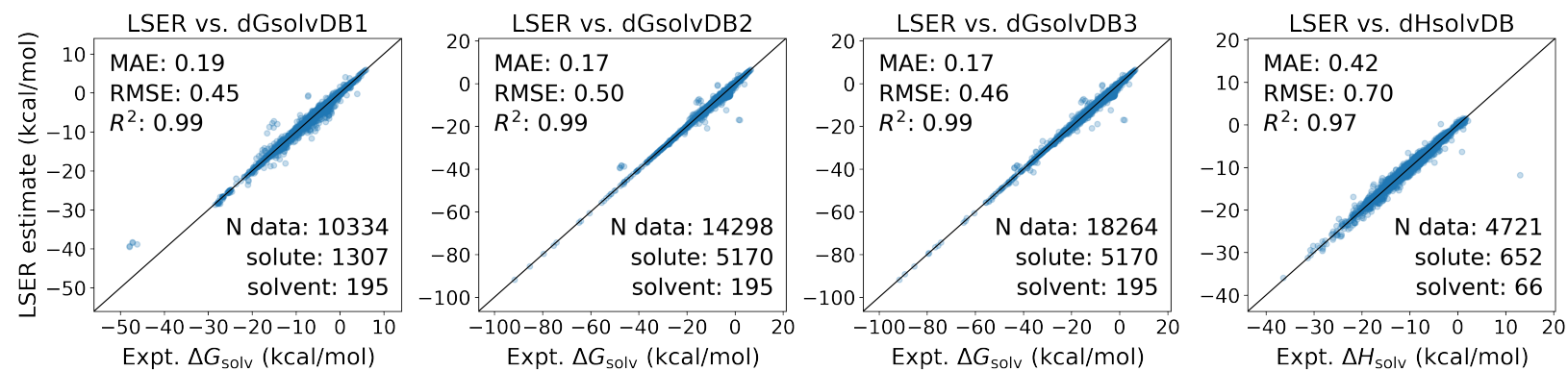

Figure S1: Comparison between the experimental $\Delta G_{\text {solv }}(298 \mathrm{~K})$ and $\Delta H_{\text {solv }}(298 \mathrm{~K})$ data and the estimations from the LSER. The LSER estimations are calculated using the Abraham solute parameter database and the fitted solvent parameters. The experimental data from dGsolvDB1, dGsolvDB2, dGsolvDB3, and dHsolvDB are compared to the LSER estimates. The mean absolute error (MAE) and root mean square error (RMSE) are reported in $\mathrm{kcal} / \mathrm{mol}$.

\section{Details on the fitted solvent parameters}

To fit Abraham ( $c, e, s, a, b, l)$ and Mintz ( $\left.c^{\prime}, e^{\prime}, s^{\prime}, a^{\prime}, b^{\prime}, l^{\prime}\right)$ solvent parameters for each solvent, we need at least 6 solvation free energy and 6 solvation enthalpy data and the solute parameters $(E, S, A, B, L)$ for the corresponding solutes. In order to avoid over-fitting, we used at least 8 data points to fit the solvent parameters. The solvent parameters are fitted using the following three regression / optimization methods: (1) ordinary least squares method from Scikit-learn package $\mathrm{e}^{\sqrt{6}}$ and (2) the dual annealing optimization and (3) robust least squares methods from SciPy package. ${ }^{7}$ After fitting three different sets of solvent parameters using the three regression methods, the set of the solvent parameters that gives the lowest prediction errors for solvation free energy or enthalpy is chosen as the final value. The 
obtained solvent parameters can be found in the XLSX file solvent_parameters.xlsx along with the compound names, SMILES, and InChI of the solvent species, the mean absolute errors of the fitted solvent parameters, and the number of data points used for fitting.

\section{Details on data sets}

\subsection{Conversion between $\log P$ and $\Delta G_{\text {solv }}$}

For the solute species with $\log K_{\mathrm{w}}$ (gas-water partition coefficient) and $\log P$ (solvent-water partition coefficient) data available, the gas-solvent partition coefficient $(\log K)$ can be calculated from Eq. S1.

$$
\log (K) \approx \log (P)+\log \left(K_{\mathrm{w}}\right)
$$

$\log K$ is subsequently converted to the solvation free energy through Eq. S2.

$$
\Delta G_{\mathrm{solv}}=-R T \ln K
$$

Note that Eq. S1 assumes that $\log P$ is measured in dry solvents, i.e. the solvents do not dissolve into one another while in contact. 


\subsection{Element and molar weight distributions of solutes in each database}

Table S1: The number of solute compounds containing each element in each data set.

\begin{tabular}{|c|c|c|c|c|c|}
\hline $\begin{array}{c}\text { N solutes containing } \\
\text { each element }\end{array}$ & SoluteDB & dGsolvDB1 & dGsolvDB2 & dGsolvDB3 & dHsolvDB \\
\hline $\mathrm{H}$ & 8252 & 11562 & 15494 & 19531 & 6024 \\
\hline $\mathrm{C}$ & 8336 & 11741 & 15708 & 19777 & 6180 \\
\hline $\mathrm{O}$ & 5232 & 5673 & 8550 & 11492 & 2709 \\
\hline $\mathrm{N}$ & 3103 & 1942 & 3603 & 5291 & 886 \\
\hline $\mathrm{S}$ & 793 & 524 & 877 & 1246 & 175 \\
\hline $\mathrm{P}$ & 123 & 70 & 140 & 194 & 9 \\
\hline $\mathrm{F}$ & 623 & 468 & 798 & 1064 & 254 \\
\hline $\mathrm{Cl}$ & 1174 & 1344 & 1946 & 2556 & 644 \\
\hline $\mathrm{Br}$ & 331 & 257 & 404 & 522 & 121 \\
\hline $\mathrm{I}$ & 116 & 146 & 189 & 228 & 58 \\
\hline
\end{tabular}
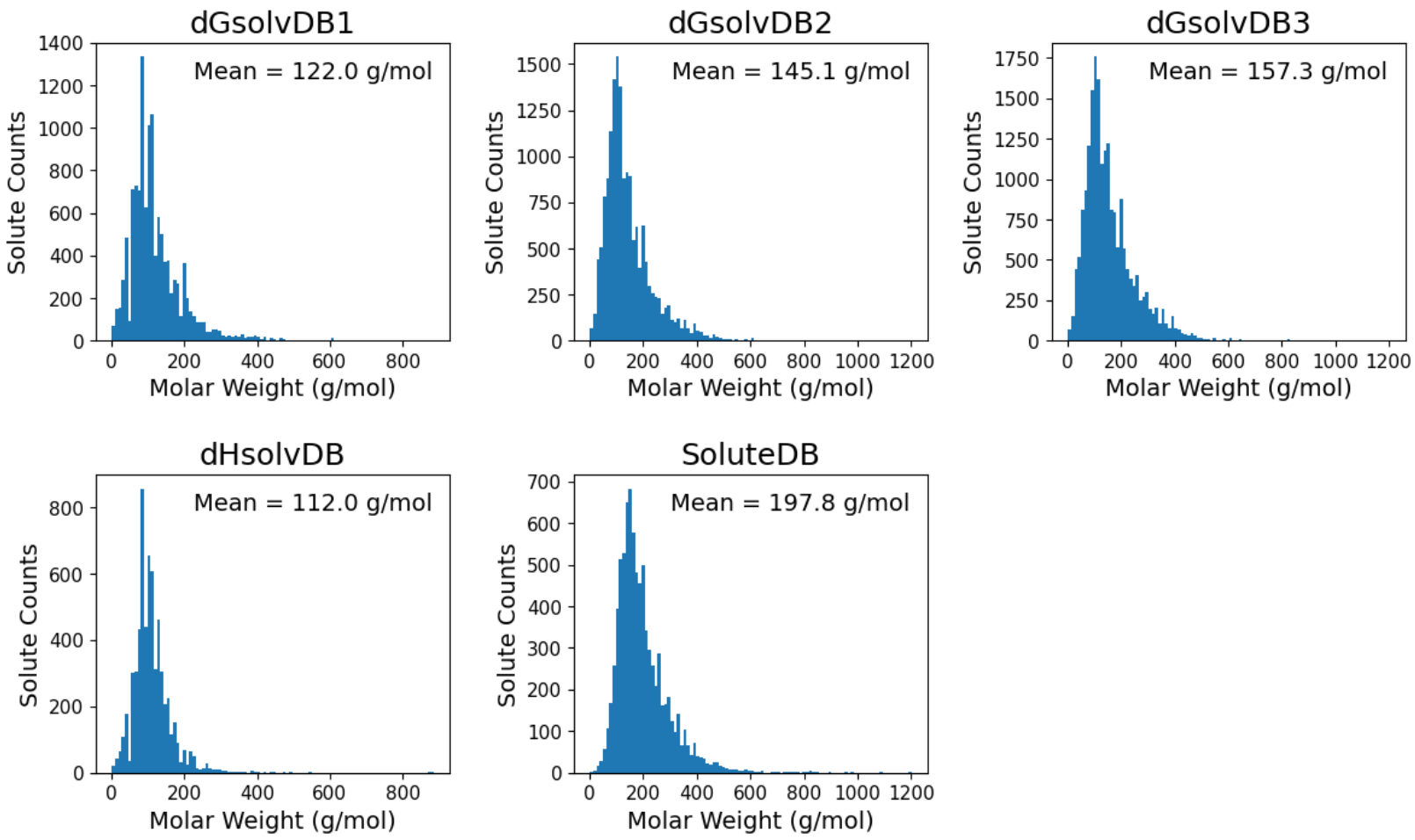

Figure S2: Molar weight distribution of solutes in each database. 


\subsection{Functional groups and scaffolds used for substructure splits}

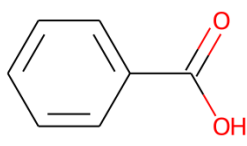

(a) Benzoic acid

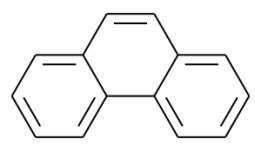

(e) Phenanthrene<smiles>[R]O[N+](=O)[O-]</smiles>

(i) Nitrate

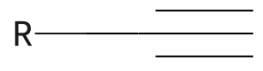

(b) 1-yne

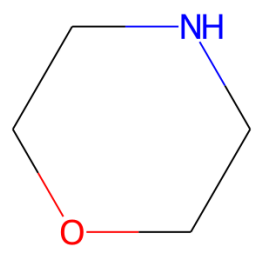

(f) Morpholine

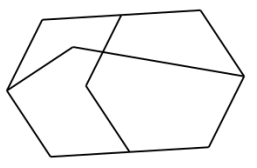

(j) Adamantane

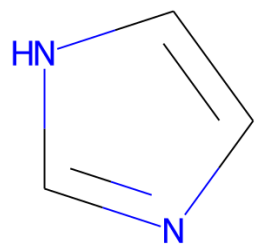

(m) Imidazole

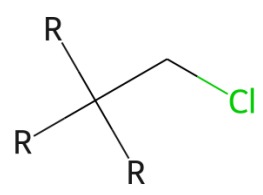

(c) Primary C-Cl

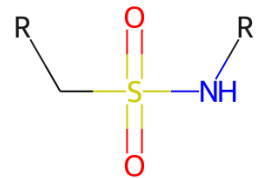

(g) Methanesulfonamide

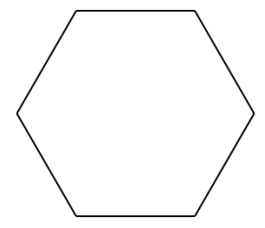

(k) Cyclohexane

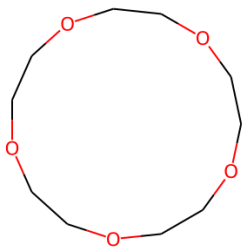

(n) 15-Crown-5

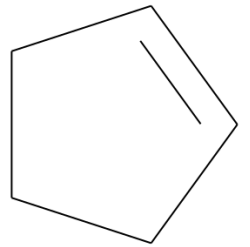

(d) Cyclopentene

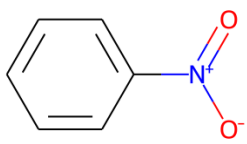

(h) Nitrobenzene

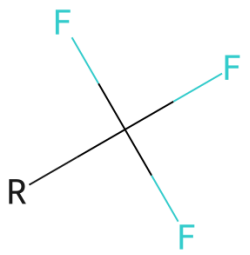

(l) Trifluoromethyl

Figure S3: Structures of the functional groups and scaffolds used for the substructure splits. 
Table S2: Names and SMARTS strings of the functional groups and scaffolds used for the solvation free energy and enthalpy substructure-based solute splits.

\begin{tabular}{|c|c|c|}
\hline data set & group name & SMARTS \\
\hline \multirow{9}{*}{$\Delta G_{\text {solv }}$} & Benzoic acid & $\mathrm{c} 1 \operatorname{ccccc} 1 \mathrm{C}(=\mathrm{O})[\mathrm{O} \& \mathrm{H} 1]$ \\
\hline & 1-yne & $\mathrm{C} \#[\mathrm{C} \& \mathrm{H} 1]$ \\
\hline & Primary $\mathrm{C}-\mathrm{Cl}$ & $\mathrm{C}[\mathrm{C} \& \mathrm{H} 2] \mathrm{Cl}$ \\
\hline & Cyclopentene & $\mathrm{C} 1=\mathrm{CCCC} 1$ \\
\hline & Phenanthrene & c1ccc2c(-,:c1)ccc1ccccc12 \\
\hline & Morpholine & C1COCCN1 \\
\hline & Methanesulfonamide & $\mathrm{NS}(=\mathrm{O})(=\mathrm{O}) \mathrm{C}$ \\
\hline & Nitrobenzene & $\operatorname{c1ccccc} 1[\mathrm{~N} \&+](=\mathrm{O})[\mathrm{O} \&-]$ \\
\hline & Nitrate & $\mathrm{O}[\mathrm{N}+]([\mathrm{O}-])=\mathrm{O}$ \\
\hline \multirow{7}{*}{$\Delta H_{\text {solv }}$} & 1-yne & $\mathrm{C} \#[\mathrm{C} \& \mathrm{H} 1]$ \\
\hline & Primary $\mathrm{C}-\mathrm{Cl}$ & $\mathrm{C}[\mathrm{C} \& \mathrm{H} 2] \mathrm{Cl}$ \\
\hline & Adamantane & $\mathrm{C} 1 \mathrm{C} 2 \mathrm{CC} 3 \mathrm{CC} 1 \mathrm{CC}(\mathrm{C} 2) \mathrm{C} 3$ \\
\hline & 15-Crown-5 & C1COCCOCCOCCOCCO1 \\
\hline & Cyclohexane & C1CCCCC1 \\
\hline & Trifluoromethyl & {$[! \mathrm{F} \& ! \mathrm{Cl} \& ! \mathrm{I} \& ! \mathrm{Br}] \mathrm{C}(-,: \mathrm{F})(-,: \mathrm{F}) \mathrm{F}$} \\
\hline & Imidazole & $\mathrm{c} 1 \mathrm{c}[\mathrm{n} \& \mathrm{H} 1] \mathrm{cn} 1$ \\
\hline
\end{tabular}

\subsection{Excluded solvents for the DirectML models}

Table S3: A list of the solvents excluded from the solvation free energy and enthalpy training sets for the DirectML models. These are the solvents used to test the DirectML models on out-of-sample solute and out-of-sample solvent pairs.

\begin{tabular}{|c|c|}
\hline$\Delta G_{\text {solv }}$ & $\Delta H_{\text {solv }}$ \\
\hline Dimethylformamide (DMF) & Ethylacetate \\
Ethylacetate & Acetonitrile \\
Chloroform & Isobutanol \\
Butanol & N-Methyl-2-pyrrolidone (NMP) \\
Chlorobenzene & Hexane \\
N-Methyl-2-pyrrolidone (NMP) & 1,2-Dichloroethane \\
Ethylether & Butylether \\
Decane & Tetrahydrofuran (THF) \\
Dioxane & \\
Acetone & \\
\hline
\end{tabular}




\subsection{Training and test sets summary}

Table S4: Training and test sets summary for solvation free energy prediction. Validation sets are randomly chosen from the training sets as needed. The data split ratio and solute split ratio both refer to the training set to test set ratio.

\begin{tabular}{|c|c|c|c|c|c|c|c|c|c|}
\hline split type & database & $\begin{array}{l}\text { data } \\
\text { split } \\
\text { ratio }\end{array}$ & $\begin{array}{l}\text { solute } \\
\text { split } \\
\text { ratio }\end{array}$ & data set & $\mathrm{n}$ data & $\begin{array}{c}\mathrm{n} \\
\text { solute }\end{array}$ & $\begin{array}{c}\mathrm{n} \\
\text { solvent }\end{array}$ & $\begin{array}{c}\text { solvent } \\
\text { water } \\
\text { data }(\%)\end{array}$ & $\begin{array}{c}\text { solvent } \\
\text { 1-octanol } \\
\text { data }(\%)\end{array}$ \\
\hline \multirow{10}{*}{ random } & \multirow[t]{2}{*}{ SoluteDB } & \multirow{2}{*}{$90: 10$} & \multirow{2}{*}{$90: 10$} & training & 7529 & 7529 & - & - & - \\
\hline & & & & testing & 837 & 837 & - & - & - \\
\hline & \multirow[t]{2}{*}{ dGsolvDB3 } & \multirow[t]{2}{*}{$89: 11$} & \multirow[t]{2}{*}{$90: 10$} & training & 18115 & 5392 & 1373 & $26.6 \%$ & $21.1 \%$ \\
\hline & & & & testing & 2138 & 599 & 284 & $26.8 \%$ & $21.3 \%$ \\
\hline & \multirow{4}{*}{$\begin{array}{c}\text { dGsolvDB3 (10 } \\
\text { solvents excluded) } \\
\text { dGsolvDB2 }\end{array}$} & \multirow[t]{2}{*}{$89: 11$} & \multirow[t]{2}{*}{$90: 10$} & training & 16902 & 5392 & 1363 & $28.5 \%$ & $22.6 \%$ \\
\hline & & & & testing & 2138 & 599 & 284 & $26.8 \%$ & $21.3 \%$ \\
\hline & & \multirow[t]{2}{*}{$89: 11$} & \multirow[t]{2}{*}{$90: 10$} & training & 14481 & 5392 & 1373 & $33.3 \%$ & $2.5 \%$ \\
\hline & & & & testing & 1699 & 599 & 284 & $33.7 \%$ & $1.9 \%$ \\
\hline & \multirow[t]{2}{*}{ dGsolvDB1 } & \multirow[t]{2}{*}{$89: 11$} & \multirow[t]{2}{*}{$90: 10$} & training & 10917 & 2160 & 1373 & $11.5 \%$ & $3.3 \%$ \\
\hline & & & & testing & 1285 & 227 & 284 & $12.3 \%$ & $2.6 \%$ \\
\hline \multirow{10}{*}{$\begin{array}{l}\text { sub- } \\
\text { structure }\end{array}$} & \multirow[t]{2}{*}{ SoluteDB } & \multirow[t]{2}{*}{$90: 10$} & \multirow[t]{2}{*}{$90: 10$} & training & 7547 & 7547 & - & - & - \\
\hline & & & & testing & 819 & 819 & - & - & - \\
\hline & \multirow[t]{2}{*}{ dGsolvDB3 } & \multirow[t]{2}{*}{$90: 10$} & \multirow[t]{2}{*}{$90: 10$} & training & 18220 & 5385 & 1391 & $26.4 \%$ & $20.8 \%$ \\
\hline & & & & testing & 2033 & 606 & 189 & $28.4 \%$ & $23.4 \%$ \\
\hline & \multirow{2}{*}{$\begin{array}{c}\text { dGsolvDB3 }(10 \\
\text { solvents excluded) }\end{array}$} & \multirow[t]{2}{*}{$89: 11$} & \multirow[t]{2}{*}{$90: 10$} & training & 17036 & 5385 & 1381 & $28.3 \%$ & $22.3 \%$ \\
\hline & & & & testing & 2033 & 606 & 189 & $28.4 \%$ & $23.4 \%$ \\
\hline & \multirow[t]{2}{*}{ dGsolvDB2 } & \multirow[t]{2}{*}{$90: 10$} & \multirow[t]{2}{*}{$90: 10$} & training & 14589 & 5385 & 1391 & $33.0 \%$ & $2.3 \%$ \\
\hline & & & & testing & 1591 & 606 & 189 & $36.3 \%$ & $3.3 \%$ \\
\hline & \multirow[t]{2}{*}{ dGsolvDB1 } & \multirow[t]{2}{*}{$90: 10$} & \multirow[t]{2}{*}{$90: 10$} & training & 11009 & 2158 & 1391 & $11.2 \%$ & $3.1 \%$ \\
\hline & & & & testing & 1193 & 229 & 189 & $15.0 \%$ & $4.4 \%$ \\
\hline
\end{tabular}

Table S5: Training and test sets summary for solvation enthalpy prediction. Validation sets are randomly chosen from the training sets as needed. The data split ratio and solute split ratio both refer to the training set to test set ratio. The percentages of solvent water and solvent 1-octanol data are not included in this table because dHsolvDB is not biased toward solvent water and 1-octanol.

\begin{tabular}{|c|c|c|c|c|c|c|c|}
\hline split type & database & $\begin{array}{l}\text { data } \\
\text { split } \\
\text { ratio }\end{array}$ & $\begin{array}{c}\text { solute } \\
\text { split } \\
\text { ratio }\end{array}$ & data set & $n$ data & $\begin{array}{c}\mathrm{n} \\
\text { solute }\end{array}$ & $\begin{array}{c}\mathrm{n} \\
\text { solvent }\end{array}$ \\
\hline \multirow{6}{*}{ random } & \multirow[t]{2}{*}{ SoluteDB } & \multirow[t]{2}{*}{$90: 10$} & \multirow[t]{2}{*}{$90: 10$} & training & 7529 & 7529 & - \\
\hline & & & & testing & 837 & 837 & - \\
\hline & \multirow[t]{2}{*}{ dHsolvDB } & \multirow[t]{2}{*}{$90: 10$} & \multirow[t]{2}{*}{$90: 10$} & training & 5679 & 1499 & 1314 \\
\hline & & & & testing & 643 & 166 & 194 \\
\hline & \multirow{2}{*}{$\begin{array}{c}\text { dHsolvDB ( } 8 \\
\text { solvents excluded) }\end{array}$} & \multirow[t]{2}{*}{$89: 11$} & \multirow[t]{2}{*}{$90: 10$} & training & 5106 & 1499 & 1306 \\
\hline & & & & testing & 643 & 166 & 194 \\
\hline \multirow{6}{*}{$\begin{array}{l}\text { sub- } \\
\text { structure }\end{array}$} & \multirow[t]{2}{*}{ SoluteDB } & \multirow[t]{2}{*}{$90: 10$} & \multirow[t]{2}{*}{$90: 10$} & training & 7510 & 7510 & - \\
\hline & & & & testing & 856 & 856 & - \\
\hline & \multirow[t]{2}{*}{ dHsolvDB } & \multirow[t]{2}{*}{$91: 9$} & \multirow[t]{2}{*}{$90: 10$} & training & 5771 & 1496 & 1289 \\
\hline & & & & testing & 551 & 169 & 228 \\
\hline & \multirow{2}{*}{$\begin{array}{c}\text { dHsolv DB (8 } \\
\text { solvents excluded) }\end{array}$} & \multirow[t]{2}{*}{$90: 10$} & \multirow[t]{2}{*}{$90: 10$} & training & 5181 & 1496 & 1281 \\
\hline & & & & testing & 551 & 169 & 228 \\
\hline
\end{tabular}




\section{Details on model methods}

\subsection{Details on the SoluteGC method}

The group values are fitted to the experimental data using the ridge regression method from Scikit-learn package ${ }^{6}$. Ridge regression is chosen as a fitting method because it gave overall the lowest error on our test sets compared to other regression methods such as ordinary least squares, lasso, and elastic net. Ridge regression ${ }^{8}$ is a linear least squares regression with L2 regularization and can help prevent overfitting. The hyperparameter of the regression is tuned using 10-fold cross-validation on each training set prepared from SoluteDB in the earlier section. The final SoluteGC model implemented in RMG is fitted using the entire data set, omitting the very small molecules where GC approach is unsuitable. All molecules with more than two heavy atoms are used to fit the group values.

As mentioned in the main text, the SoluteGC method is built within RMG, ${ }^{9}$ which has the group contribution (GC) method for gas phase thermochemistry, and much of the SoluteGC framework follows that of the RMG's gas phase GC method. In RMG, the structure of a functional group is represented as an adjacency list, a graph representation that uses atoms as nodes and bonds as edges. Atom and bond types are specified in the adjacency list to help creating both general and specific functional groups. Detailed explanation on the adjacency list, atom types, and bond types can be found in the RMG documentation at http: //reactionmechanismgenerator.github.io/RMG-Py/reference/molecule/ adjlist.html and http://reactionmechanismgenerator.github.io/ RMG-Py/users/rmg/database/introduction.html\#introdatabase

The functional groups in the SoluteGC method are categorized into 6 different types as described in Table 3 of the main text. Each of the group types is stored as a separate file in RMG-database under RMG-database/input/solvation/groups/, which can be found at https://github.com/ReactionMechanismGenerator/ RMG-database/tree/master/input/solvation/groups. The name of each file name is listed in Table $\mathbf{S 6}$ below.

Table S6: The group categories and their corresponding file names in RMG-database. The group files are located under RMG-database/input/solvation/groups/ in RMGdatabase.

\begin{tabular}{|c|c|}
\hline Group Category & File Name in RMG-database \\
\hline AC-regular & group.py \\
\hline AC-halogen & halogen.py \\
\hline RSC-ring & ring.py \\
\hline RSC-polycyclic & polycyclic.py \\
\hline LDI-cyclic & longDistanceInteraction_cyclic.py \\
\hline LDI-noncyclic & longDistanceInteraction_noncyclic.py \\
\hline
\end{tabular}

In each group file listed in Table 56 , the functional groups are organized in a hierarchical tree structure: general groups are located at top nodes as parent groups and more specific 
groups are placed underneath the parent groups as children. The group structures are defined so that children groups are always subsets of their parent groups structurally. For each atom or ring cluster found in a molecule, a group is identified by descending down the group hierarchical tree until the most specific functional group is matched. The matched group has its corresponding fitted group values, which in this case are the solute parameters, $E, S$, $A, B$ and $L$, and also the number of data used to fit each group value.

\subsection{SoluteML and DirectML model hyperparameters}

For each model, the hyperparameters are selected based on a search algorithm that includes optimization of the neural network for different hyperparameter combinations. The search procedure combines the algorithms available in the software package Hyperopt ${ }^{10}$ with manual intervention to balance the size of the neural networks with the gain in accuracy. The hyperparameters that are optimized include the depth and hidden size of the D-MPNN, the number of layers and hidden size of the neural network for property prediction, the learning rates, and the batch size. An overview of the selected hyperparameters for the different models is given in Table $\mathrm{S7}$.

Table S7: Hyperparameters used for the SoluteML and DirectML models. The DirectML model uses the same hyperparameters for the prediction of $\Delta G_{\text {solv }}(298 \mathrm{~K})$ and $\Delta H_{\text {solv }}(298 \mathrm{~K})$. MPNN: massage-passing neural network, FFN: feed-forward neural network for property prediction.

\begin{tabular}{|c|c|c|}
\hline Hyperparameter & SoluteML & DirectML \\
\hline MPNN depth & 3 & 3 \\
\hline MPNN hidden size & 400 & 400 \\
\hline MPNN bias & No & No \\
\hline FFN number of layers & 4 & 3 \\
\hline FFN hidden size & 1200 & Yes \\
\hline FFN bias & Yes & LeakyReLU \\
\hline Activation function & LeakyReLU & 0.0 \\
\hline Dropout & 0.0 & 50 \\
\hline Batch size & 50 & 4 \\
\hline Warm-up epochs & 4 & 200 \\
\hline Max epochs & 250 & $(5 \mathrm{e}-4,5 \mathrm{e}-4,1 \mathrm{e}-6)$ \\
\hline
\end{tabular}

The ML models are trained with 5-fold cross-validation. For each of the different splits, the test set is pre-defined as detailed in the main text Section 2.2. During the 5-fold crossvalidation, a different random validation set of $10 \%$ is generated and distinguished from the training set to determine at which iteration (or epoch) to stop training the model. Within each fold, an ensemble of 5 different models is generated by Glorot initialization of the model parameters ${ }^{11}$ with different seeds. All models are used to make predictions for the pre-defined test set. The predictions of each individual model are averaged to calculate the prediction of 
the model ensemble. Additionally, the variance on the predictions of the individual models is used as a measure for the model uncertainty (i.e. epistemic uncertainty) on the individual data points.

\section{Results - comparison of the DirectML models trained on different dGsolvDB data sets}

The performance of the DirectML models that are trained and validated on three different solvation free energy data sets (dGsolvDB1, dGsolvDB2, dGsolvDB3) are compared in this section. As explained in the main text Section 2.1, the size of the data set increases from dGsolvDB1 to dGsolvDB3, including more solutes, but the solvents become more skewed towards water and 1-octanol as $\log K_{\mathrm{w}}$ and $\log P$ data are added. To select the best performing model, only overlapping test data between the three DirectML models trained with the three data sets are compared. Because each data set is the subset of the next larger data set $(\mathrm{dGsolvDB} 1 \subset \mathrm{dGsolvDB} 2 \subset \mathrm{dGsolvDB} 3)$, the overlapping test data only include the $\Delta G_{\text {solv }}$ data from dGsolvDB1 and do not include in-house $\log K_{\mathrm{w}}$ and $\log P$ data.

The test errors of the three models on the overlapping test set data are shown on the parity plots in Figure S4. For a random split, the performance of the three models is very similar. The model trained on dGsolvDB3 gives the lowest MAE of $0.29 \mathrm{kcal} / \mathrm{mol}$, which is only $0.02 \mathrm{kcal} / \mathrm{mol}$ lower than the model trained on dGsolvDB1. The difference between the models is a bit more pronounced in the substructure split. The model trained on dGsolvDB3 gives the lowest MAE of $0.81 \mathrm{kcal} / \mathrm{mol}$, which is $0.06 \mathrm{kcal} / \mathrm{mol}$ lower than the model trained on dGsolvDB1. The result indicates that adding both $\log K_{\mathrm{w}}$ and $\log P$ data to the training and validation data set slightly improves solvation energy predictions compared to training and validating the models on only solvation free energy data. Yet, considering that the total number of data and the number of solutes in dGsolvDB3 are nearly twice as many as those in dGsolvDB1, the improvement is not as significant as expected.

In Figure S5, a similar comparison is done using the complete (10\%) test set of each respective data set rather than only the overlapping data. In this case, the model trained, validated, and tested on dGsolvDB1 gives the lowest MAE of $0.31 \mathrm{kcal} / \mathrm{mol}$ compared to 0.40 $\mathrm{kcal} / \mathrm{mol}$ for the model trained, validated, and tested on dGsolvDB3 for a random split. For the substructure split, the difference is less significant with the lowest MAE of $0.87 \mathrm{kcal} / \mathrm{mol}$ for dGsolvDB1 closely followed by $0.89 \mathrm{kcal} / \mathrm{mol}$ for dGsolvDB3. If the PMAE is compared instead, the dGsolvDB1 and dGsolvDB3 models both have the lowest PMAE of $4.9 \%$ for the random split and the dGsolvDB3 model has the lowest PMAE of $9.2 \%$ for the substructure split compared to the PMAE of $10.1 \%$ for the dGsolvDB1 model.

These observations are in line with the conclusions made in earlier work by Vermeire and Green ${ }^{12}$. The model performance improves with an increasing amount of data in the training and validation set; however, the extent to which the model performance can be assessed is limited by the experimental (or aleatoric) uncertainty in the test set. For the same test set, the performance of the three models on a random split is similar since the aleatoric limit of assessing the performance on that test set is reached. For the substructure split, there is still a slight improvement in performance observed by the addition of more data to the training and validation set. For the comparison against the complete $(10 \%)$ test set of each data 
(a) Random Split

Test data $=1285$, solute $=227$, solvent $=284$, mean $=-6.34 \mathrm{kcal} / \mathrm{mol}$
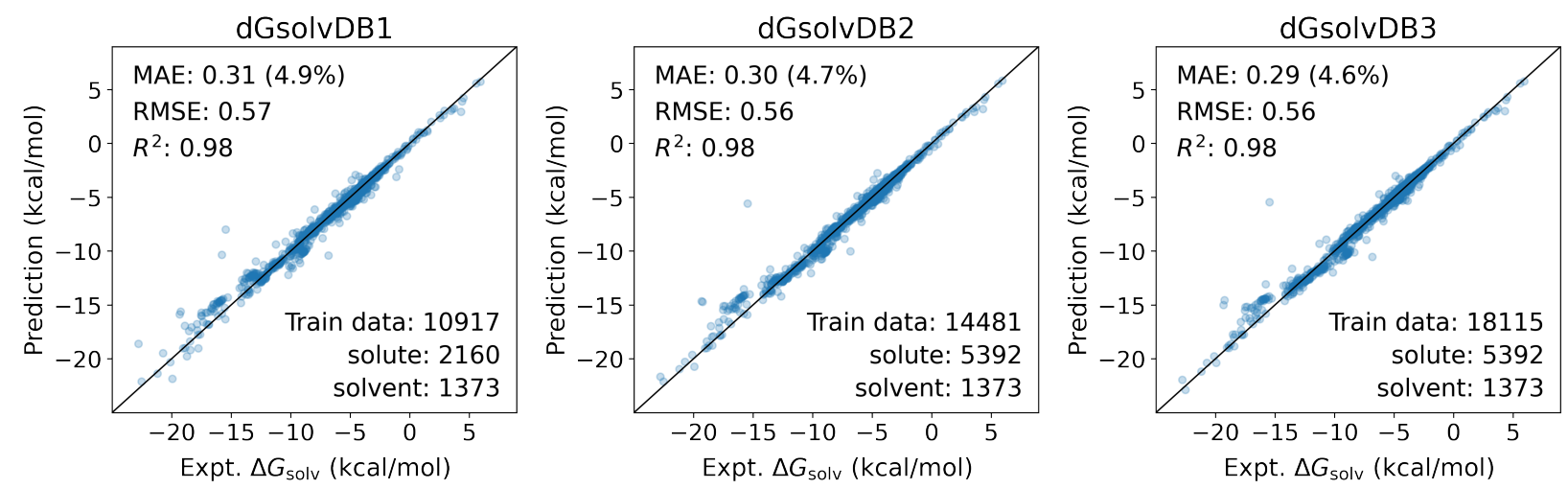

(b) Substructure Split

Test data $=1193$, solute $=229$, solvent $=189$, mean $=-8.57 \mathrm{kcal} / \mathrm{mol}$
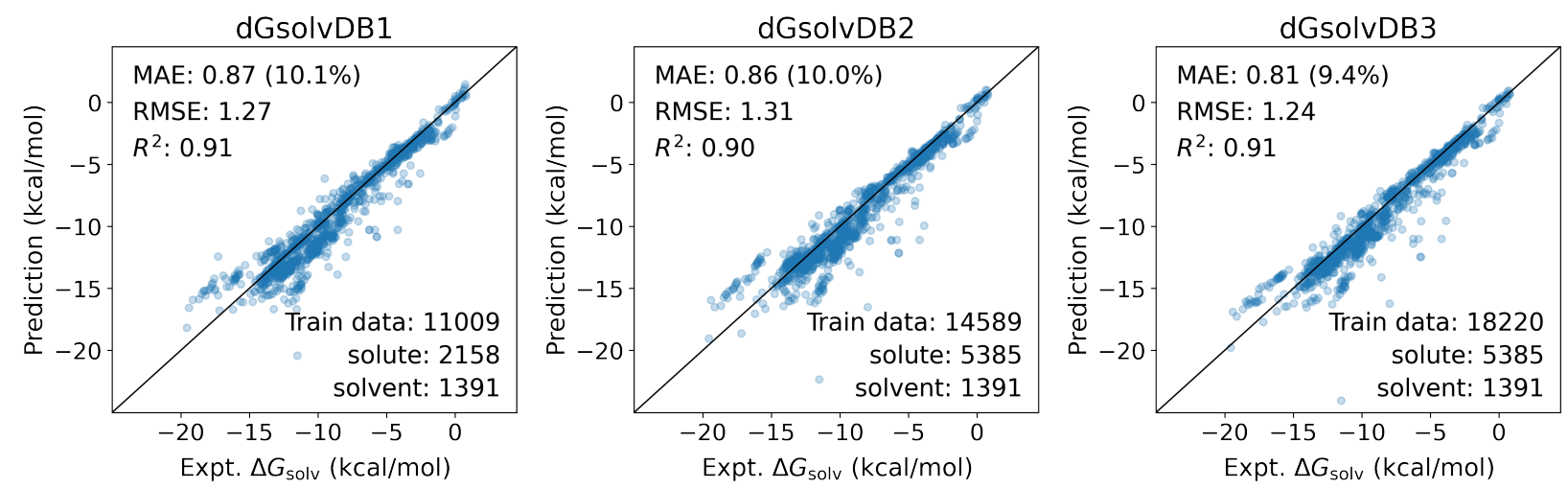

Figure S4: Parity plots of the DirectML models trained and validated using 3 different solvation free energy data sets (dGsolvDB1, dGsolvDB2, and dGsolvDB3) that differ in the type and amount of solvation data considered. The plots only show the overlapping test data in each split type. The MAE and RMSE are in kcal/mol, and the PMAE is given in parenthesis. Information on the training and test data are given on the figures together with the overall errors. Note that the test sets are prepared using random and substructure-based solute splits, and therefore none of the test set solutes appear in the training and validation sets.

set in Figure $\mathrm{S5}$, it is expected that the test splits of the data sets containing $\log K_{\mathrm{w}}$ and $\log P$ data have a higher associated experimental uncertainty. The experimental uncertainty in solvation free energies of neutral compounds is typically estimated as $0.2 \mathrm{kcal} / \mathrm{mol} \frac{13[14}{\text {, }}$ but the solvation free energies measured in water $\left(\log K_{\mathrm{w}}\right)$ often have higher uncertainties ${ }^{15}$, which we believe are due to larger magnitudes of the values and disagreements between data reported by different sources. The conversion of $\log P$ data to solvation free energies also comes along with an additional uncertainty because of the assumption that solvents are not in contact with one another. As a result, the model trained and validated on dGsolvDB1 seems to have better performance if we compare the MAE evaluated on the complete test set. 
(a) Random Split
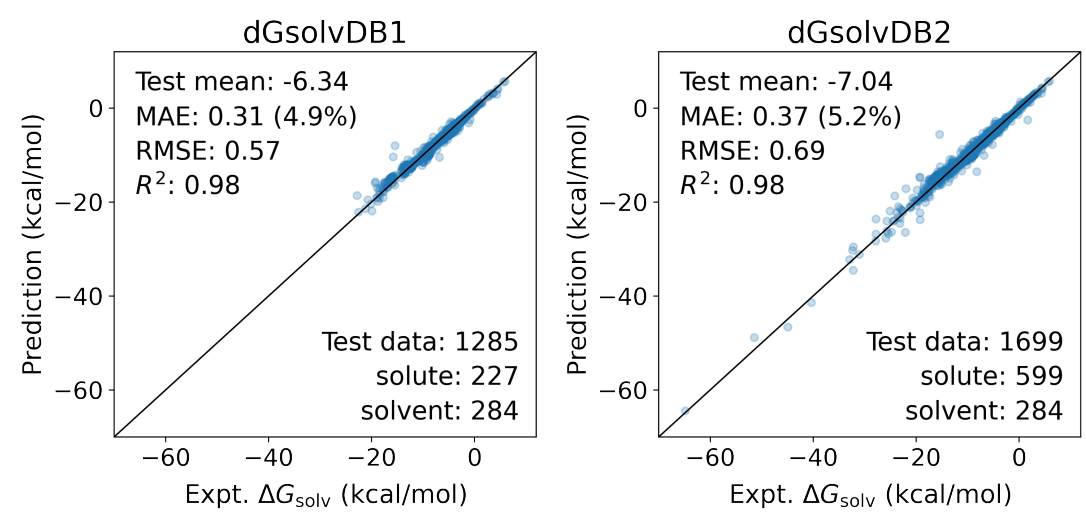

(b) Substructure Split
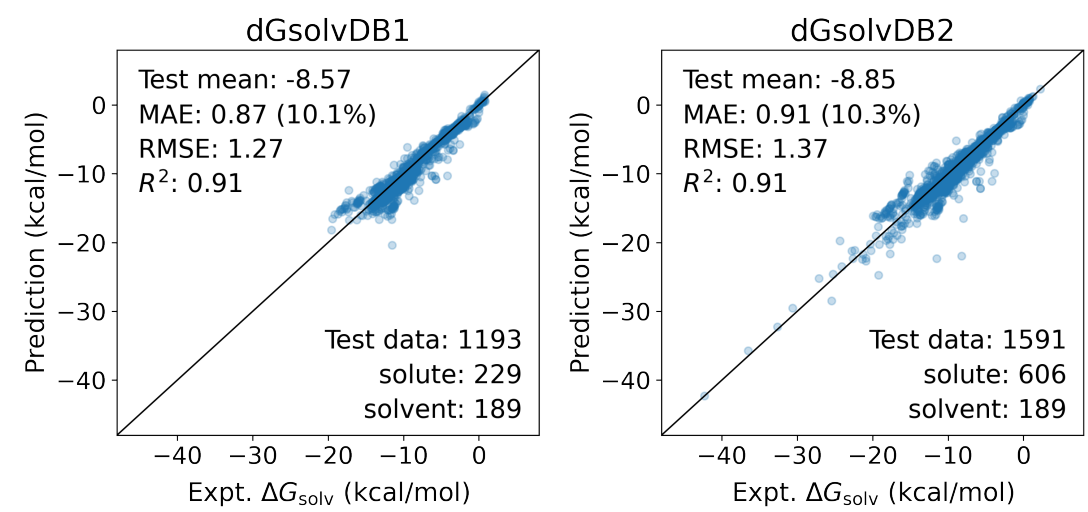
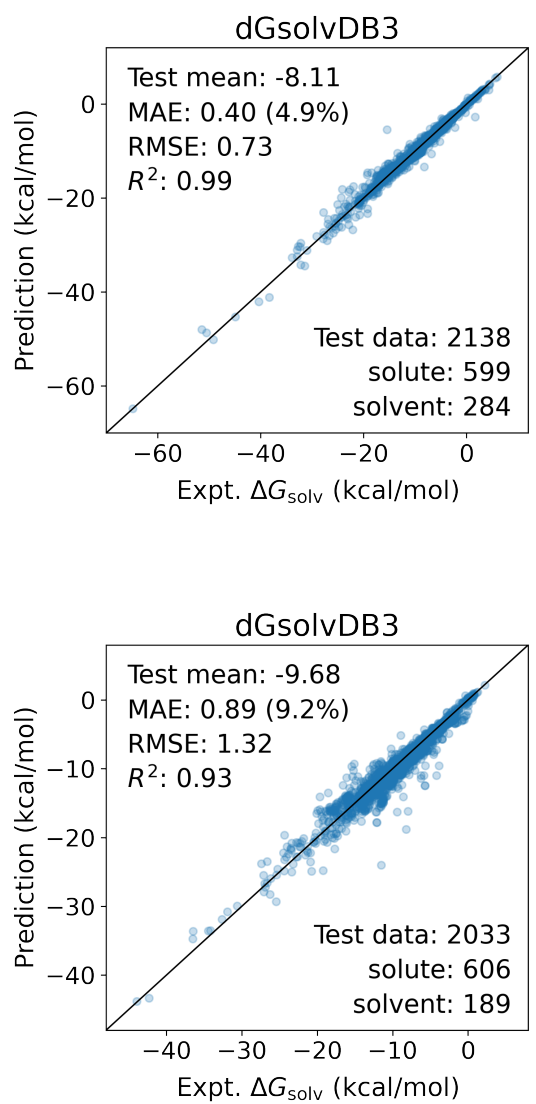

Figure S5: Parity plots of the DirectML models trained and validated using 3 different solvation free energy data sets (dGsolvDB1, dGsolvDB2, and dGsolvDB3) that differ in the type and amount of solvation data considered. The plots show the results using all of the individual model's test set data. The MAE, RMSE, and test mean are in $\mathrm{kcal} / \mathrm{mol}$, and the PMAE is given in parenthesis. Information on the number of total data (test data), solutes, and solvents in the test set is given on the figures together with the overall errors. Note that the test sets are prepared using random and substructure-based solute splits, and therefore none of the test set solutes appear in the training and validation sets.

However, this conclusion cannot be directly drawn because of (1) the additional uncertainty in the other test sets and (2) the different range of the magnitudes of solvation free energies in the test set data. The latter is compensated for by comparing the PMAE of the different models instead of the MAE or RMSE.

Overall, including more data in the training and validation set is beneficial as it lowers the prediction error when assessed on the overlapping test set data, and thus dGsolvDB3 is chosen as the optimal solvation free energy data set. We also compare the solvent-wise test errors of the three DirectML models in Figure S6, and it is found that having data bias towards two opposing solvents like water and 1-octanol does not cause any particularly high errors in other solvents. However, since the accuracy of the three models is not significantly different, it is possible that different data splits or data sizes can yield contrasting results. 

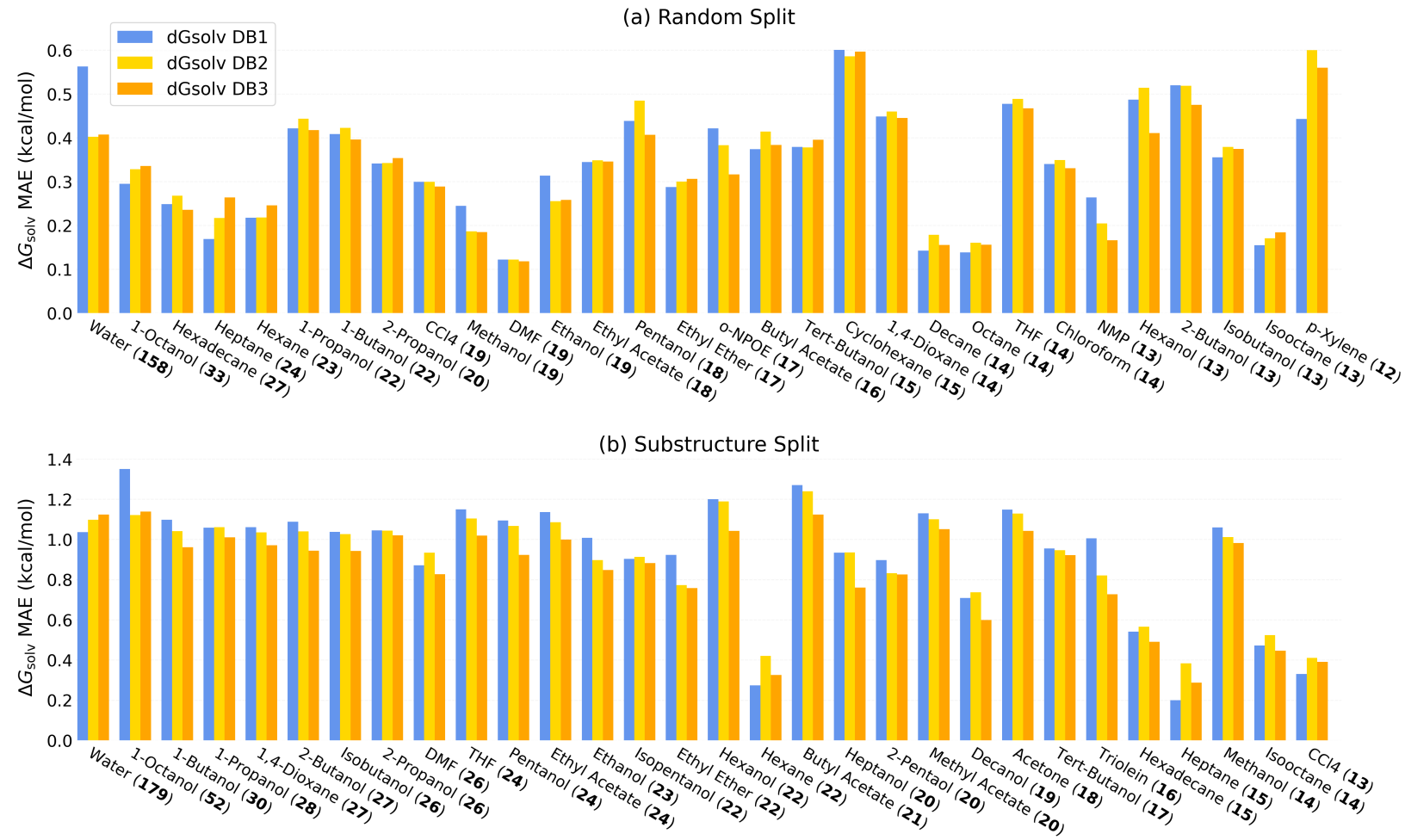

Figure S6: The MAE for the prediction of $\Delta G_{\text {solv }}(298 \mathrm{~K})$ on the 30 most frequently appearing solvents in each split type. The performance of the DirectML models trained using 3 different solvation free energy data sets (dGsolvDB1, dGsolvDB, and dGsolvDB3) is compared only using the overlapping test data. The number of solvent data in each test set is given in parenthesis in bold. (CCl4: carbon tetrachloride, DMF: dimethylformamide, o-NPOE: 2nitrophenyl octyl ether, THF: tetrahydrofuran, NMP: N-methyl-2-pyrrolidone)

\section{Results - solvent-wise error comparison of the three prediction approaches}

The solvent-wise errors of the three models are compared for the 30 most frequently appearing solvents in each test set as shown in Figure S7. The figure shows the results on the unseen solutes in the trained solvents. For the majority of the solvents for $\Delta G_{\text {solv }}(298 \mathrm{~K})$ prediction, the DirectML model performs similar to or better than the SoluteML model, and the SoluteGC model gives the highest errors. In the random split set of $\Delta G_{\text {solv }}(298 \mathrm{~K})$, the SoluteML model performs better than the DirectML model for non-polar solvents such as alkanes, carbon tetrachloride $(\mathrm{CCl} 4)$, isooctane, and p-xylene. However, the DirectML model gives the best predictions for the same non-polar solvents in the substructure split set. Similarly, the SoluteGC and SoluteML models have much higher errors for dimethylformamide (DMF), ethyl acetate, and ethyl ether than the DirectML model in the random split, but their performance is similar to the DirectML model in the substructure split for the same solvents (with exception of the SoluteML model for DMF). No clear correlation between the model performance and the types of solvents is found from the results as there are no specific types of solvents that a certain model always underperforms or outperforms. 


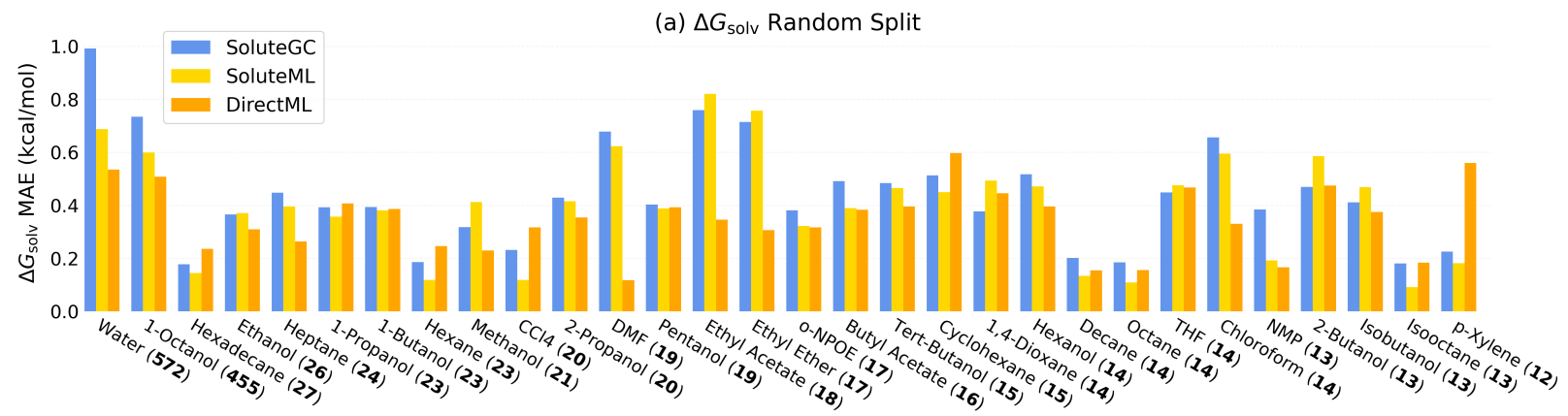

(b) $\Delta G_{\text {solv }}$ Substructure Split

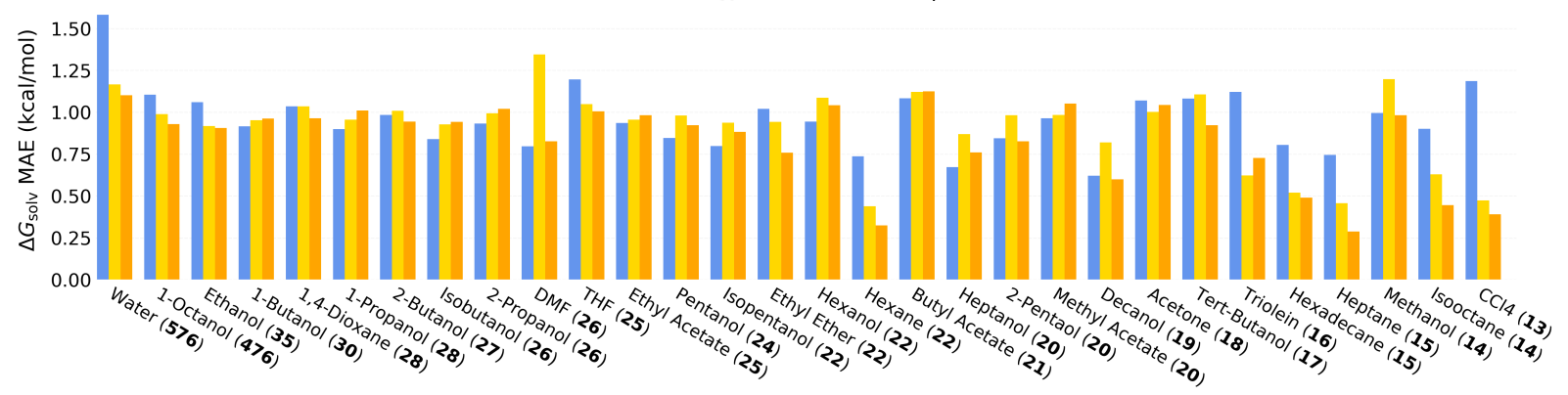

(c) $\Delta H_{\text {solv }}$ Random Split
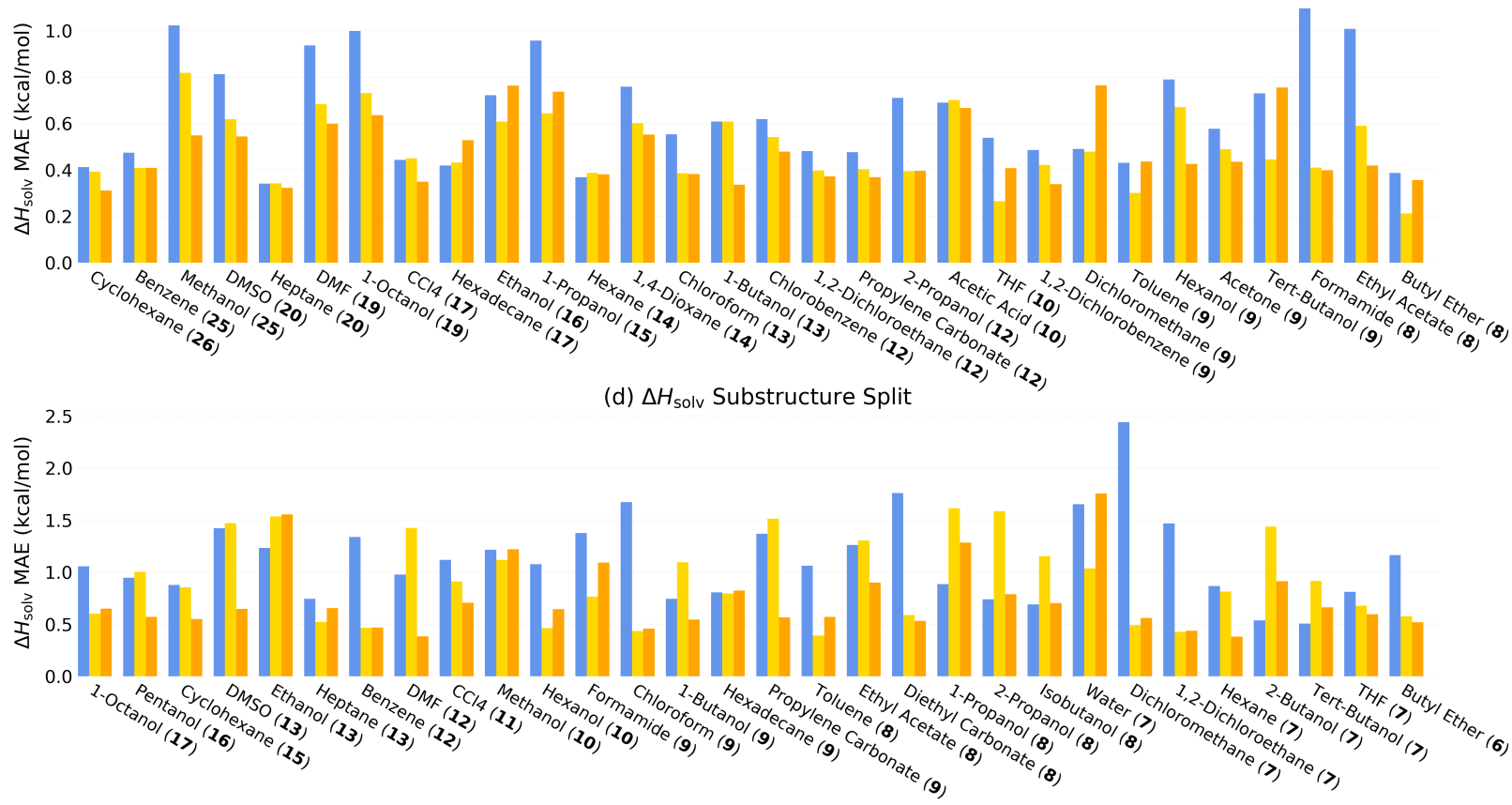

Figure S7: The MAE for the prediction of $\Delta G_{\text {solv }}(298 \mathrm{~K})$ and $\Delta H_{\text {solv }}(298 \mathrm{~K})$ on the 30 most frequently appearing solvents in each test set. The performance of the different models is compared only using the overlapping test data. The number of solvent data in each test set is given in parenthesis in bold. All solvents are included in the training sets of the DirectML models. (CCl4: carbon tetrachloride, DMF: dimethylformamide, o-NPOE: 2-nitrophenyl octyl ether, THF: tetrahydrofuran, NMP: N-methyl-2-pyrrolidone, DMSO: dimethyl sulfoxide) 
It is also noted that the three models have very similar error scales for most of the solvents.

Similar to the prediction of $\Delta G_{\text {solv }}(298 \mathrm{~K})$, the DirectML model performs better than the other two models for the majority of the solvents in the prediction of $\Delta H_{\text {solv }}(298 \mathrm{~K})$ in Figure S7. Some exceptions include hexadecane, ethanol, dichloromethane, and tert-butanol for which the SoluteML model gives much lower errors than the DirectML model in the random split, but the SoluteML and DirectML models have similar prediction accuracy for the same solvents in the substructure split. The three models have different levels of accuracy for many solvents for the $\Delta H_{\text {solv }}(298 \mathrm{~K})$ prediction in the substructure split compared to the $\Delta G_{\text {solv }}(298 \mathrm{~K})$ prediction. 


\section{Results - supplementary figures}

This section includes the supplementary figures relevant to each result subsection of the main text. It includes the parity plots of the various models on all of their test set data, the parity plots of the average model predictions, and the results of the SoluteGC and SoluteML models on the solute parameter predictions. In the parity plots, MAE, RMSE, and test mean represent the mean absolute error, root-mean-square error, and average experimental value in the test set, respectively.

\subsection{Comparison of the three prediction approaches}

\subsubsection{Performance on unseen solutes for all test data}

(a) $\Delta G_{\text {solv }}$ Random Split
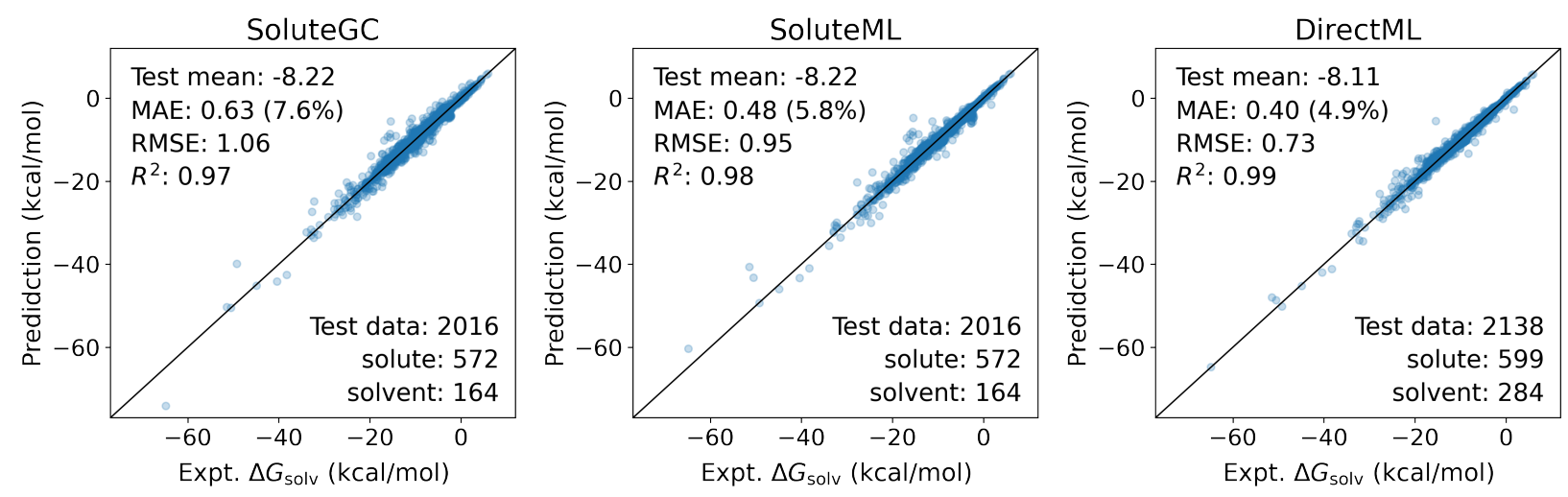

(b) $\Delta G_{\text {solv }}$ Substructure Split
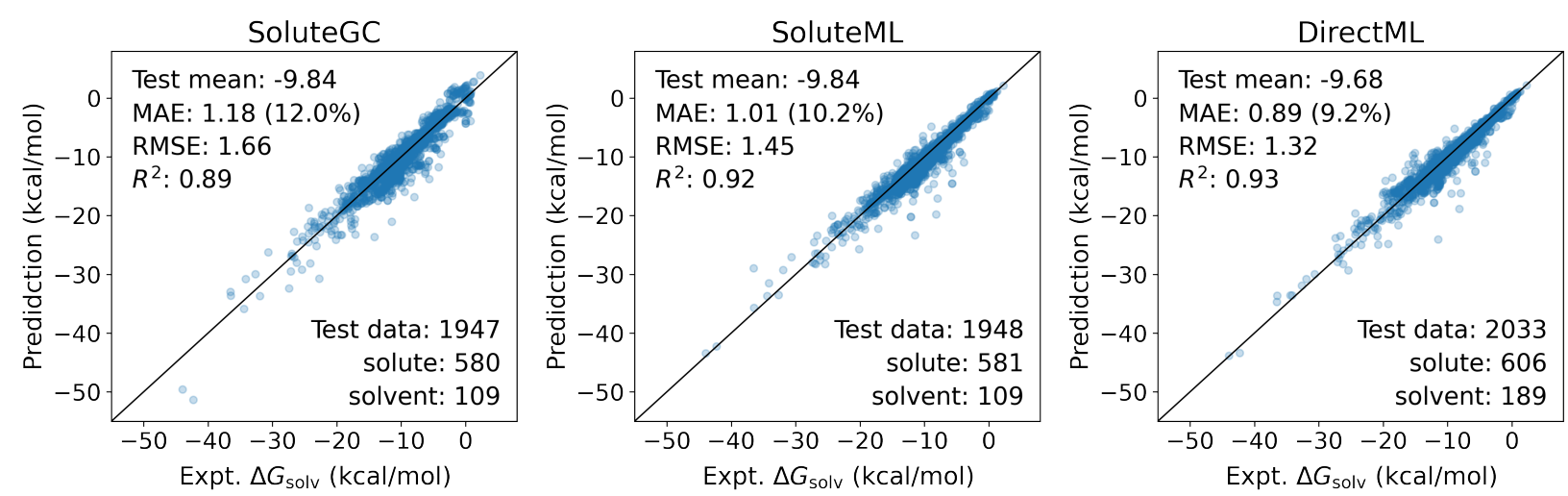

Figure S8: Parity plots for experimental and predicted $\Delta G_{\text {solv }}(298 \mathrm{~K})$ on the $10 \%$ test sets. The plots show the results using all of the individual model's test set data. The three different models are tested on unseen solutes for the random and substructure splits. The MAE, RMSE, and test mean are in $\mathrm{kcal} / \mathrm{mol}$, and the PMAE is given in parenthesis. Information on the number of total data (test data), solutes, and solvents in the test set is given on the figures together with the overall errors. 
(a) $\Delta H_{\text {solv }}$ Random Split

SoluteGC

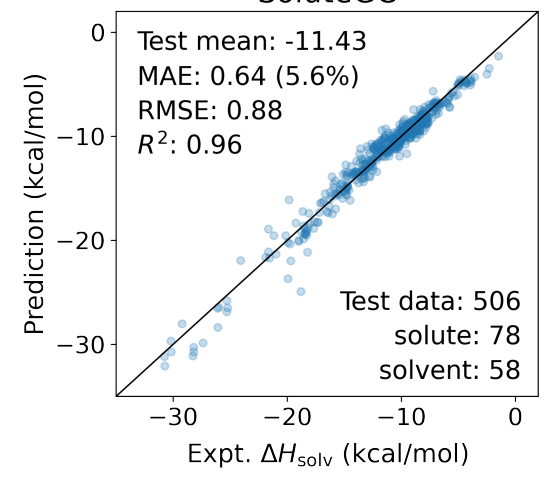

SoluteGC

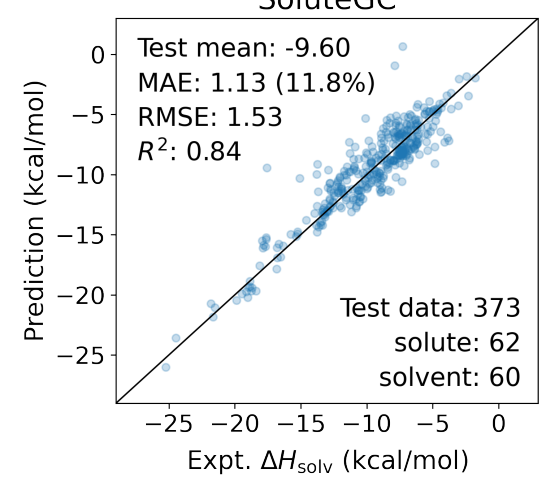

SoluteML

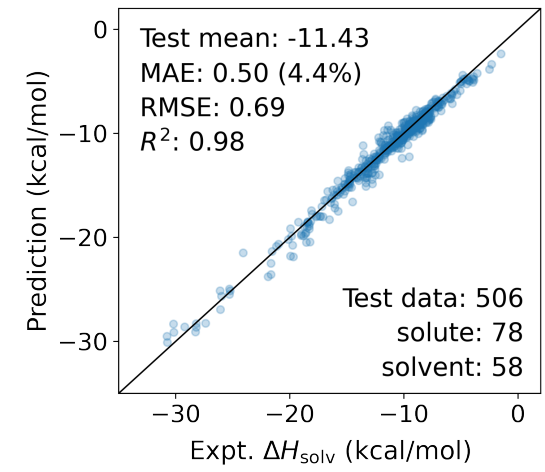

(b) $\Delta H_{\text {solv }}$ Substructure Split

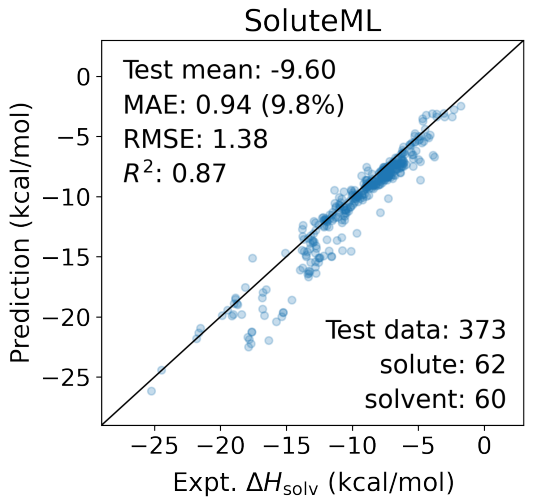

DirectML

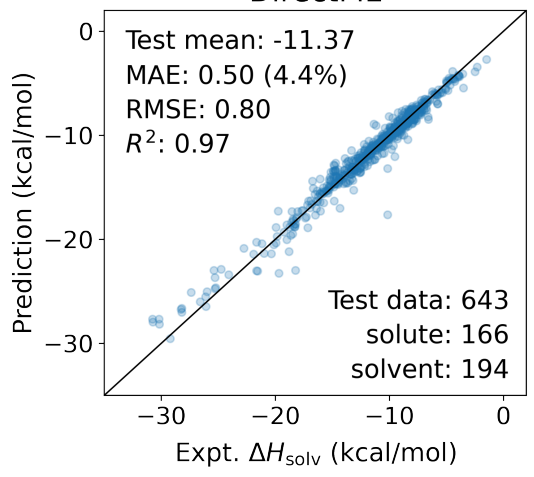

DirectML

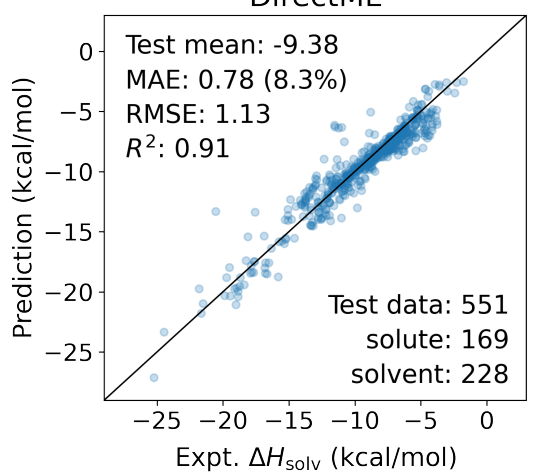

Figure S9: Parity plots for experimental and predicted $\Delta H_{\text {solv }}(298 \mathrm{~K})$ on the $10 \%$ test sets. The plots show the results using all of the individual model's test set data. The three different models are tested on unseen solutes for the random and substructure splits. The MAE, RMSE, and test mean are in $\mathrm{kcal} / \mathrm{mol}$, and the PMAE is given in parenthesis. Information on the number of total data (test data), solutes, and solvents in the test set is given on the figures together with the overall errors. 


\subsection{Parity plot of the average model prediction on unseen solutes}

(a) Average prediction of 2 models (SoluteML, DirectML)
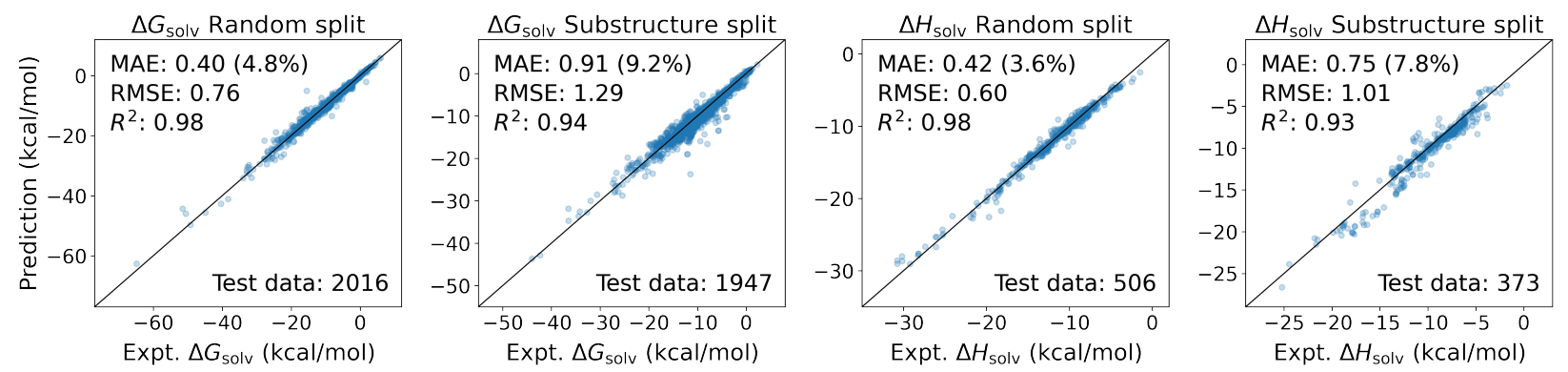

(b) Average prediction of 3 models (SoluteGC, SoluteML, DirectML)
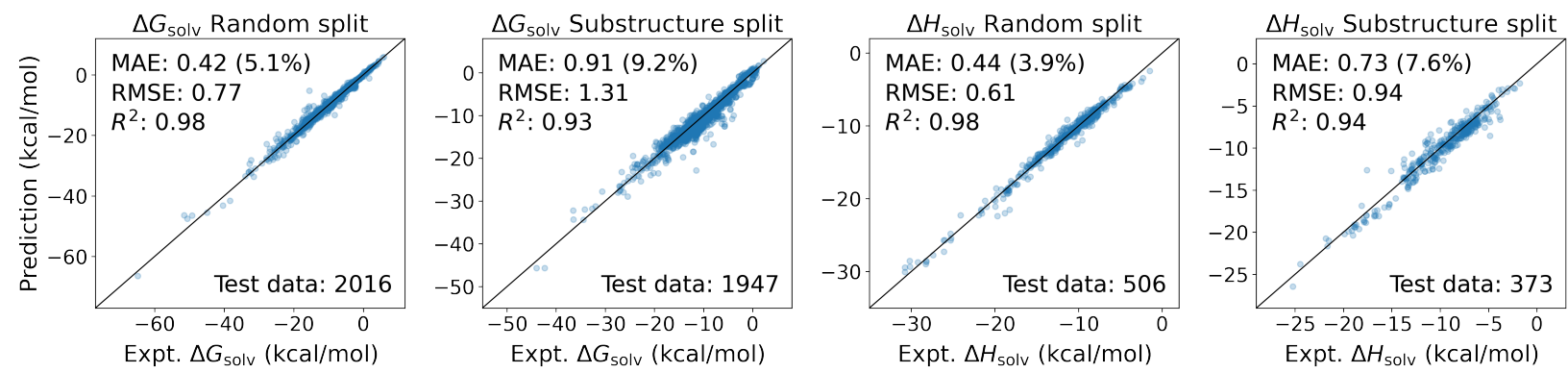

Figure S10: Parity plots between the experimental data and average predictions of the two different models (SoluteML, DirectML) and the three different models (SoluteGC, SoluteML, DirectML) on the overlapping test data. The parity plots are for $\Delta G_{\text {solv }}(298 \mathrm{~K})$ and $\Delta H_{\text {solv }}(298 \mathrm{~K})$ predictions tested on pairs of unseen solutes and trained solvents for the random and substructure splits. The MAE and RMSE are in $\mathrm{kcal} / \mathrm{mol}$, and the PMAE is given in parenthesis. The number of test data are also given on the figures along with the test errors. The plots only show the overlapping test data in each split type. 


\subsubsection{Performance on unseen solutes for solute parameter predictions}

(a) Solute Parameter Prediction for the $\Delta G_{\text {solv }}$ Random Split Test Set
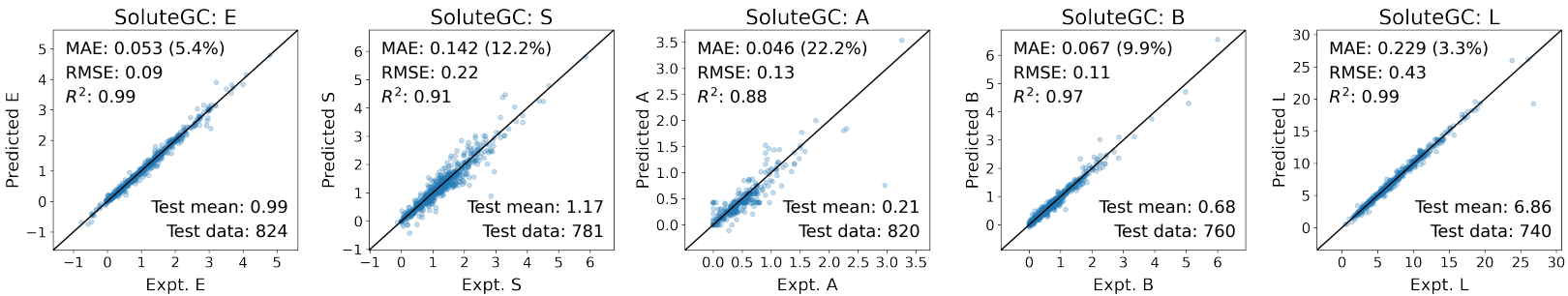

SoluteML: E
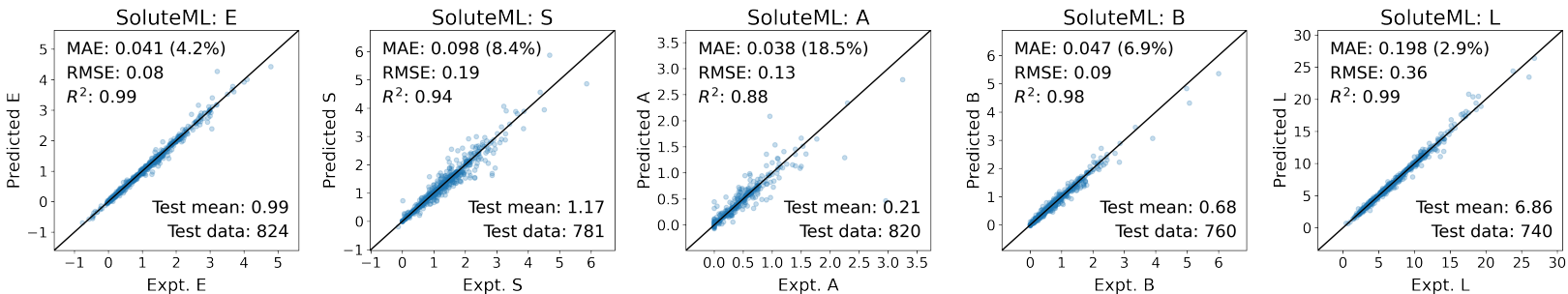

(b) Solute Parameter Prediction for the $\Delta G_{\text {solv }}$ Substructure Split Test Set
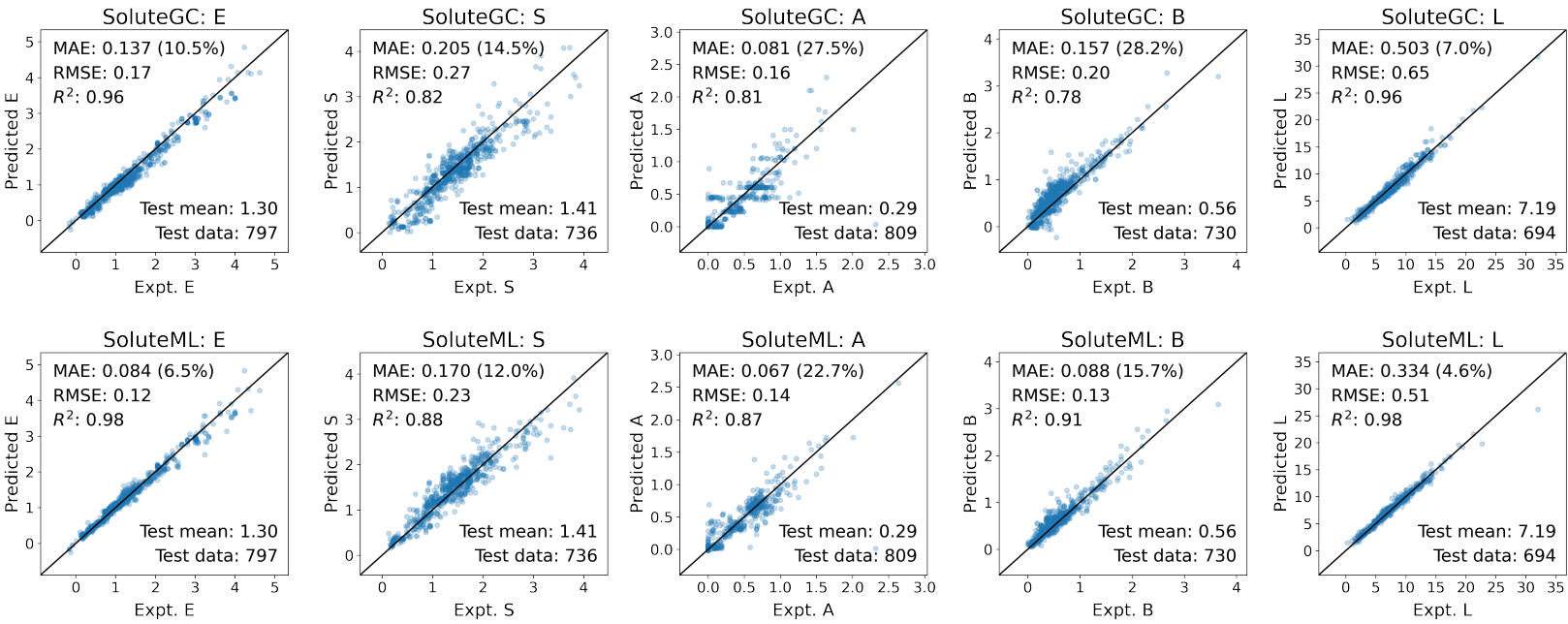

Figure S11: Parity plots for experimental and predicted solute parameters $(E, S, A, B, L)$ on the $10 \%$ test sets prepared for $\Delta G_{\text {solv }}(298 \mathrm{~K})$ prediction comparison. The plots show the results using all of the individual model's test set data. The SoluteGC and SoluteML are tested on unseen solutes for the random and substructure splits. The MAE, RMSE, and test mean have no units, and the PMAE is given in parenthesis. The number of test data (test data) is given on the figures together with the overall errors. 
(a) Solute Parameter Prediction for the $\Delta H_{\text {solv }}$ Random Split Test Set

SoluteGC: E

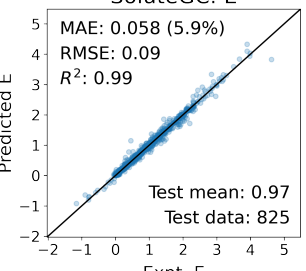

Expt. E

SoluteML: E

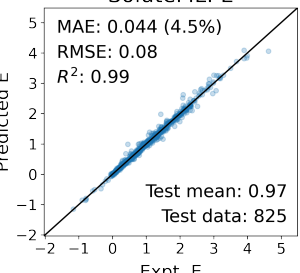

Expt. E

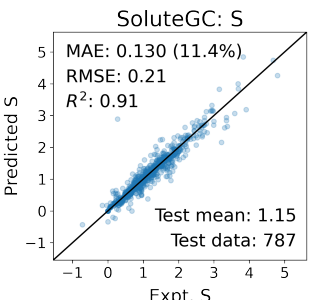

oluteML:

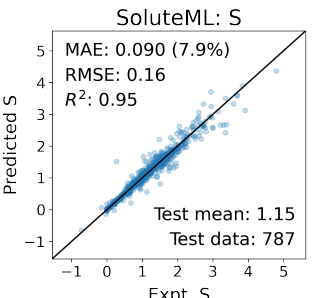

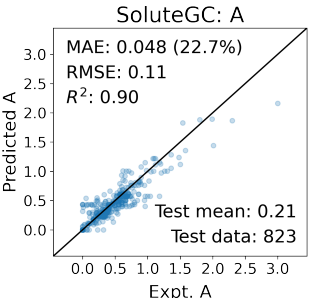

Expt. A

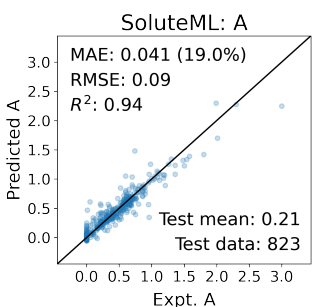

SoluteGC: B

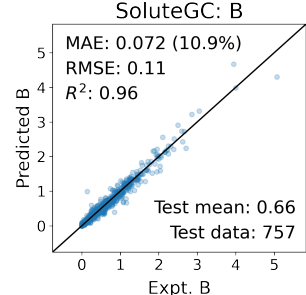

SoluteML: B

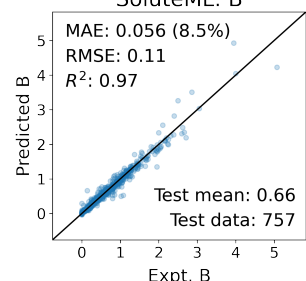

SoluteGC: L

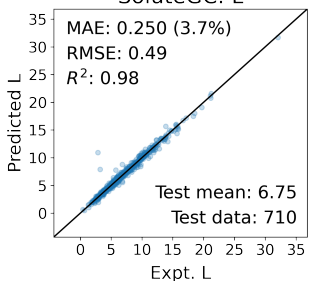

SoluteML:

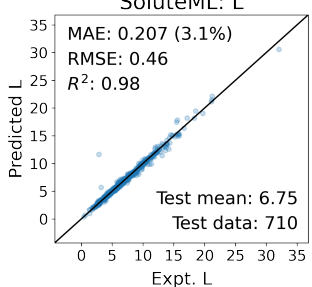

(b) Solute Parameter Prediction for the $\Delta H_{\text {solv }}$ Substructure Split Test Set

SoluteGC: $\mathrm{E}$

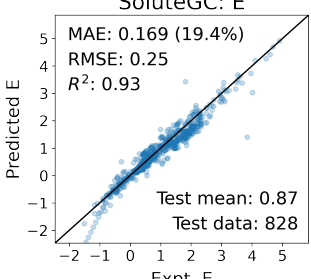

Expt. E

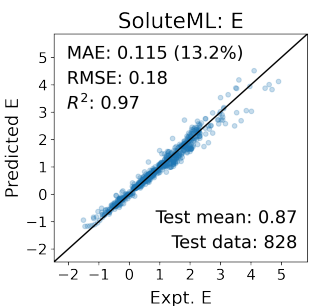

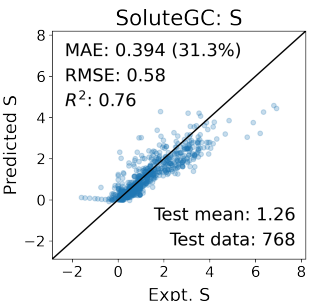

SoluteML: S

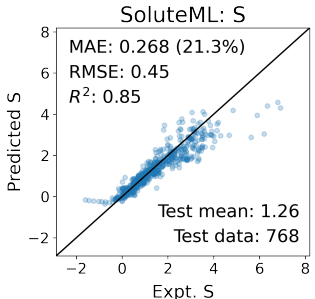

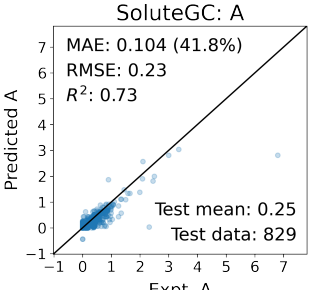

SoluteGC: B
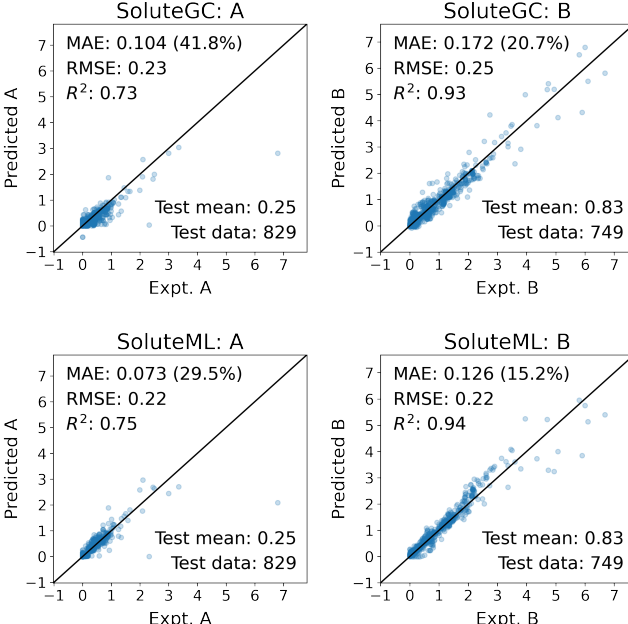

SoluteML: B

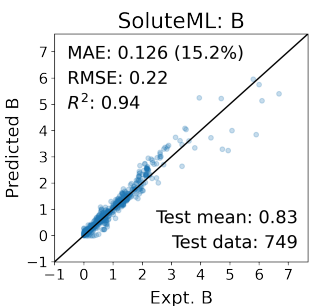

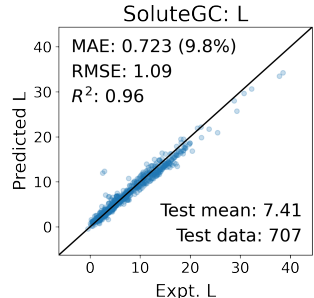

SoluteML: L

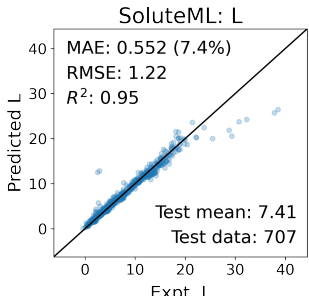

Figure S12: Parity plots for experimental and predicted solute parameters $(E, S, A, B, L)$ on the $10 \%$ test sets prepared for $\Delta H_{\text {solv }}(298 \mathrm{~K})$ prediction comparison. The plots show the results using all of the individual model's test set data. The SoluteGC and SoluteML are tested on unseen solutes for the random and substructure splits. The MAE, RMSE, and test mean have no units, and the PMAE is given in parenthesis. The number of test data (test data) is given on the figures together with the overall errors. 


\subsection{Comparison to existing methods}

\subsubsection{COSMO-RS prediction results}
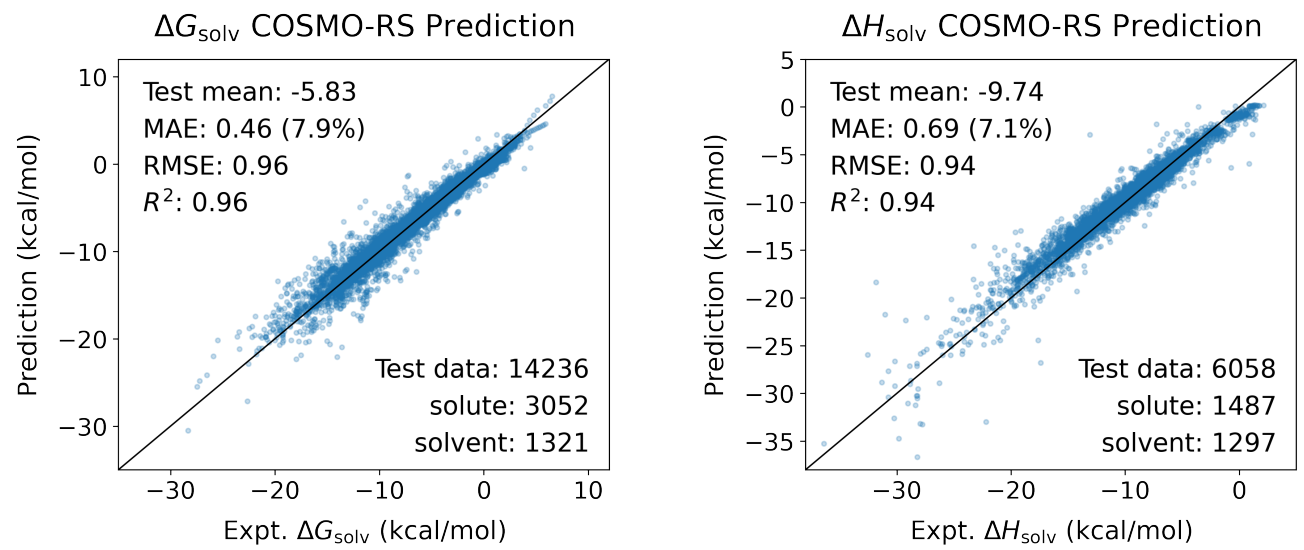

Figure S13: Parity plots of the COSMO-RS BP/TZVPD-FINE method for $\Delta G_{\text {solv }}(298 \mathrm{~K})$ and $\Delta H_{\text {solv }}(298 \mathrm{~K})$ predictions. The MAE, RMSE, and test mean are all in $\mathrm{kcal} / \mathrm{mol}$, and the PMAE is given in parenthesis. The number of total data, solutes, and solvents tested for the COSMO-RS method is given on the figures together with the overall errors. 


\subsubsection{UFZ-LSER GC prediction results}
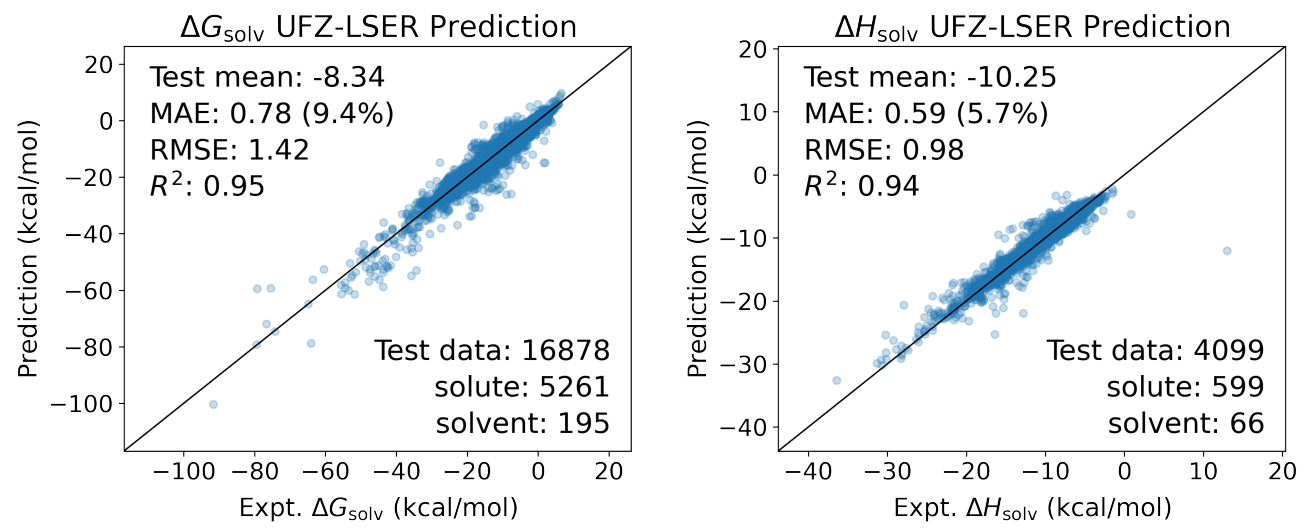

Figure S14: Parity plots of the UFZ-LSER GC method for $\Delta G_{\text {solv }}(298 \mathrm{~K})$ and $\Delta H_{\text {solv }}(298 \mathrm{~K})$ predictions. The MAE, RMSE, and test mean are all in $\mathrm{kcal} / \mathrm{mol}$, and the PMAE is given in parenthesis. The number of total data, solutes, and solvents tested for the UFZ-LSER method is given on the figures together with the overall errors.
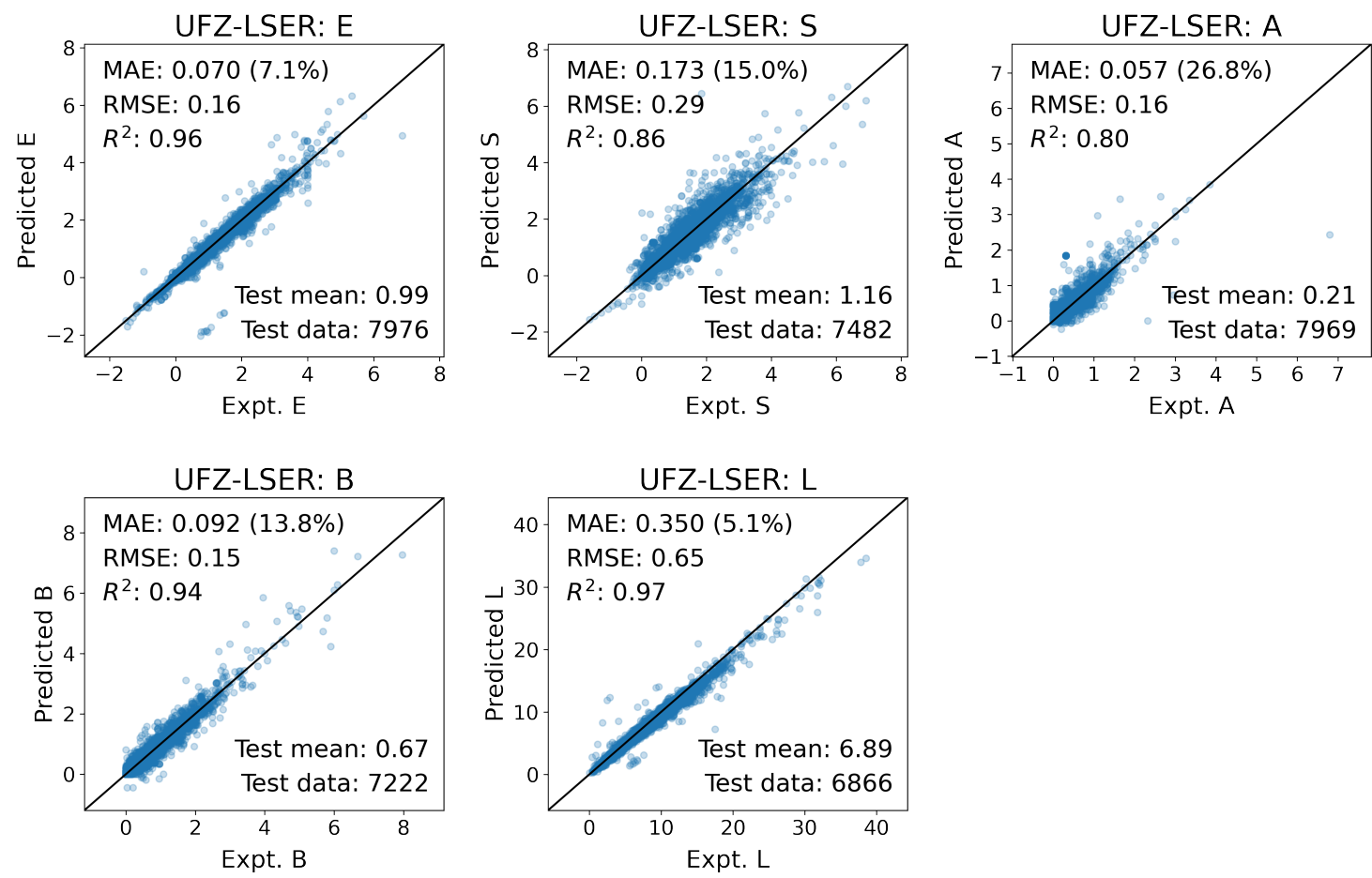

Figure S15: Parity plots of the UFZ-LSER GC method for the solute parameter $(E, S, A$, $B, L)$ predictions. The MAE, RMSE, and test mean have no units, and the PMAE is given in parenthesis. The number of test data (test data) is given on the figures together with the overall errors. 


\subsubsection{Final model training error}

Table S8: The errors of the final SoluteGC, SoluteML, and DirectML models trained on all data. The MAE and RMSE are in $\mathrm{kcal} / \mathrm{mol}$, and the number of each data set used for error calculation $\left(N_{\text {data }}\right)$ is presented.

\begin{tabular}{|c|c|c|c|c|c|c|c|}
\hline target & method & $\begin{array}{c}\text { method } \\
\text { type }\end{array}$ & model detail & MAE & RMSE & $N_{\text {data }}$ & note \\
\hline$\Delta G_{\text {solv }}$ & DirectML (final) & ML & $\begin{array}{c}5 \text {-fold random CV test error } \\
\text { (trained on all data) }\end{array}$ & 0.25 & 0.54 & 203 & $a$ \\
\cline { 2 - 8 } & DirectML (final) & ML & $\begin{array}{c}5 \text {-fold random CV validation error } \\
\text { (trained on all data) }\end{array}$ & 0.26 & 0.52 & 810 & $a$ \\
\cline { 2 - 8 } & DirectML (final) & ML & $\begin{array}{c}5 \text {-fold random CV training error } \\
\text { (trained on all data) }\end{array}$ & 0.06 & 0.12 & 19240 & $a$ \\
\cline { 2 - 8 } & SoluteML (final) & ML & $\begin{array}{c}\text { trained on all } \\
\text { solute parameter data }\end{array}$ & 0.27 & 0.67 & 18655 & $b$ \\
\cline { 2 - 8 } & SoluteGC (final) & GC & $\begin{array}{c}\text { trained on all } \\
\text { solute parameter data }\end{array}$ & 0.65 & 1.10 & 16884 & $c$ \\
\hline \multirow{5}{*}{$\Delta H_{\text {solv }}$} & DirectML (final) & ML & $\begin{array}{c}5 \text {-fold random CV test error } \\
\text { (trained on all data) }\end{array}$ & 0.49 & 0.77 & 64 & $a$ \\
\cline { 2 - 8 } & DirectML (final) & ML & $\begin{array}{c}5 \text {-fold random CV validation error } \\
\text { (trained on all data) }\end{array}$ & 0.44 & 0.73 & 253 & $a$ \\
\cline { 2 - 8 } & DirectML (final) & ML & $\begin{array}{c}5 \text {-fold random CV training error } \\
\text { (trained on all data) }\end{array}$ & 0.25 & 0.39 & 6005 & $a$ \\
\cline { 2 - 8 } & SoluteML (final) & ML & $\begin{array}{c}\text { trained on all } \\
\text { solute parameter data }\end{array}$ & 0.44 & 0.74 & 4731 & $b$ \\
\cline { 2 - 8 } & SoluteGC (final) & GC & $\begin{array}{c}\text { trained on all } \\
\text { solute parameter data }\end{array}$ & 0.61 & 0.98 & 4099 & $c$ \\
\hline
\end{tabular}

${ }^{a}$ The error is calculated using 5 -fold cross validation with $95 \%$ training, $4 \%$ validation, and $1 \%$ test sets. ${ }^{b}$ RDKit is not able to generate some of the 2D molecular features for molecular hydrogen, and therefore, molecular hydrogen is omitted from the final SoluteML error calculations. ${ }^{c}$ Only the solutes with more than two heavy atoms are used to train the SoluteGC model. 


\section{References}

[1] Heller, S. R.; McNaught, A.; Pletnev, I.; Stein, S.; Tchekhovskoi, D. InChI, the IUPAC International Chemical Identifier. J. Cheminf. 2015, 7, 23.

[2] Swain, M. PubChemPy. 2013; https://pypi.org/project/PubchemPy/1.0/, (accessed 2021-12-20).

[3] Swain, M. CIRpy. 2016; https://pypi.org/project/CIRpy/, (accessed 202112-20).

[4] Landrum, G. RDKit: Open-Source Cheminformatics. 2006; http://www.rdkit. org/, (accessed 2021-12-20).

[5] Grinberg Dana, A.; Ranasinghe, D.; Wu, H.; Grambow, C.; Dong, X.; Johnson, M.; Goldman, M.; Liu, M.; Green, W. H. ARC - Automated Rate Calculator, version 1.1.0. https://github.com/ReactionMechanismGenerator/ARC, 2019; (accessed 2021-12-20).

[6] Pedregosa, F.; Varoquaux, G.; Gramfort, A.; Michel, V.; Thirion, B.; Grisel, O.; Blondel, M.; Prettenhofer, P.; Weiss, R.; Dubourg, V.; Vanderplas, J.; Passos, A.; Cournapeau, D.; Brucher, M.; Perrot, M.; Duchesnay, E. Scikit-learn: Machine Learning in Python. J. Mach. Learn. Res. 2011, 12, 2825-2830.

[7] Virtanen, P.; Gommers, R.; Oliphant, T. E.; Haberland, M.; Reddy, T.; Cournapeau, D.; Burovski, E.; Peterson, P.; Weckesser, W.; Bright, J.; van der Walt, S. J.; Brett, M.; Wilson, J.; Millman, K. J.; Mayorov, N.; Nelson, A. R. J.; Jones, E.; Kern, R.; Larson, E.; Carey, C. J.; Polat, I.; Feng, Y.; Moore, E. W.; VanderPlas, J.; Laxalde, D.; Perktold, J.; Cimrman, R.; Henriksen, I.; Quintero, E. A.; Harris, C. R.; Archibald, A. M.; Ribeiro, A. H.; Pedregosa, F.; van Mulbregt, P.; SciPy 1.0 Contributors, SciPy 1.0: Fundamental Algorithms for Scientific Computing in Python. Nat. Methods 2020, 17, $261-272$.

[8] Hoerl, A. Application of ridge analysis to regression problems. Chem. Eng. Prog. 1962, $58,54-59$.

[9] Gao, C. W.; Allen, J. W.; Green, W. H.; West, R. H. Reaction Mechanism Generator: Automatic construction of chemical kinetic mechanisms. Comput. Phys. Commun. 2016, 203, 212-225.

[10] Bergstra, J.; Yamins, D.; Cox, D. D. Making a Science of Model Search: Hyperparameter Optimization in Hundreds of Dimensions for Vision Architectures. Proceedings of the 30th International Conference on Machine Learning. Atlanta, Georgia, 2013; pp I-115 to I-23.

[11] Glorot, X.; Bengio, Y. Understanding the difficulty of training deep feedforward neural networks. J. Mach. Learn. Res. 2010, 9, 249-256. 
[12] Vermeire, F. H.; Green, W. H. Transfer learning for solvation free energies: From quantum chemistry to experiments. Chem. Eng. J. 2021, 418, 129307.

[13] Thompson, J. D.; Cramer, C. J.; Truhlar, D. G. New universal solvation model and comparison of the accuracy of the SM5.42R, SM5.43R, C-PCM, D-PCM, and IEFPCM continuum solvation models for aqueous and organic solvation free energies and for vapor pressures. J. Phys. Chem. A 2004, 108, 6532-6542.

[14] Nicholls, A.; Mobley, D. L.; Guthrie, J. P.; Chodera, J. D.; Bayly, C. I.; Cooper, M. D.; Pande, V. S. Predicting small-molecule solvation free energies: An informal blind test for computational chemistry. J. Med. Chem. 2008, 51, 769-779.

[15] Guthrie, J. P. A blind challenge for computational solvation free energies: Introduction and overview. J. Phys. Chem. B 2009, 113, 4501-4507. 